\title{
Large particle segregation, transport and accumulation in granular free-surface flows
}

\author{
J. M. N. T. GRA Y ${ }^{1} \dagger$ AND B. P. KOKELAAR \\ ${ }^{1}$ School of Mathematics and Manchester Centre for Nonlinear Dynamics, University of Manchester, \\ Manchester M13 9PL, UK \\ ${ }^{2}$ Earth and Ocean Sciences Department, University of Liverpool, Liverpool L69 3GP, UK
}

(Received 19 October 2009; revised 18 December 2009; accepted 27 December 2009)

Particle size segregation can have a significant feedback on the motion of many hazardous geophysical mass flows such as debris flows, dense pyroclastic flows and snow avalanches. This paper develops a new depth-averaged theory for segregation that can easily be incorporated into the existing depth-averaged structure of typical models of geophysical mass flows. The theory is derived by depth-averaging the segregation-remixing equation for a bi-disperse mixture of large and small particles and assuming that (i) the avalanche is always inversely graded and (ii) there is a linear downslope velocity profile through the avalanche depth. Remarkably, the resulting 'large particle transport equation' is very closely related to the segregation equation from which it is derived. Large particles are preferentially transported towards the avalanche front and then accumulate there. This is important, because when this is combined with mobility feedback effects, the larger less mobile particles at the front can be continuously shouldered aside to spontaneously form lateral levees that channelize the flow and enhance run-out. The theory provides a general framework that will enable segregation-mobility feedback effects to be studied in detail for the first time. While the large particle transport equation has a very simple representation of the particle size distribution, it does a surprisingly good job of capturing solutions to the full theory once the grains have segregated into inversely graded layers. In particular, we show that provided the inversely graded interface does not break it has precisely the same solution as the full theory. When the interface does break, a concentration shock forms instead of a breaking size segregation wave, but the net transport of large particles towards the flow front is exactly the same. The theory can also model more complex effects in small-scale stratification experiments, where particles may either be brought to rest by basal deposition or by the upslope propagation of a granular bore. In the former case the resulting deposit is normally graded, while in the latter case it is inversely graded. These completely opposite gradings in the deposit arise from a parent flow that is inversely graded, which raises many questions about how to interpret geological deposits.

\section{Introduction}

Granular avalanches are very effective at sorting particles by size, through a combination of 'kinetic sieving' and 'squeeze expulsion' (Middleton 1970; Savage \&

$\dagger$ Email address for correspondence: nico.gray@manchester.ac.uk 


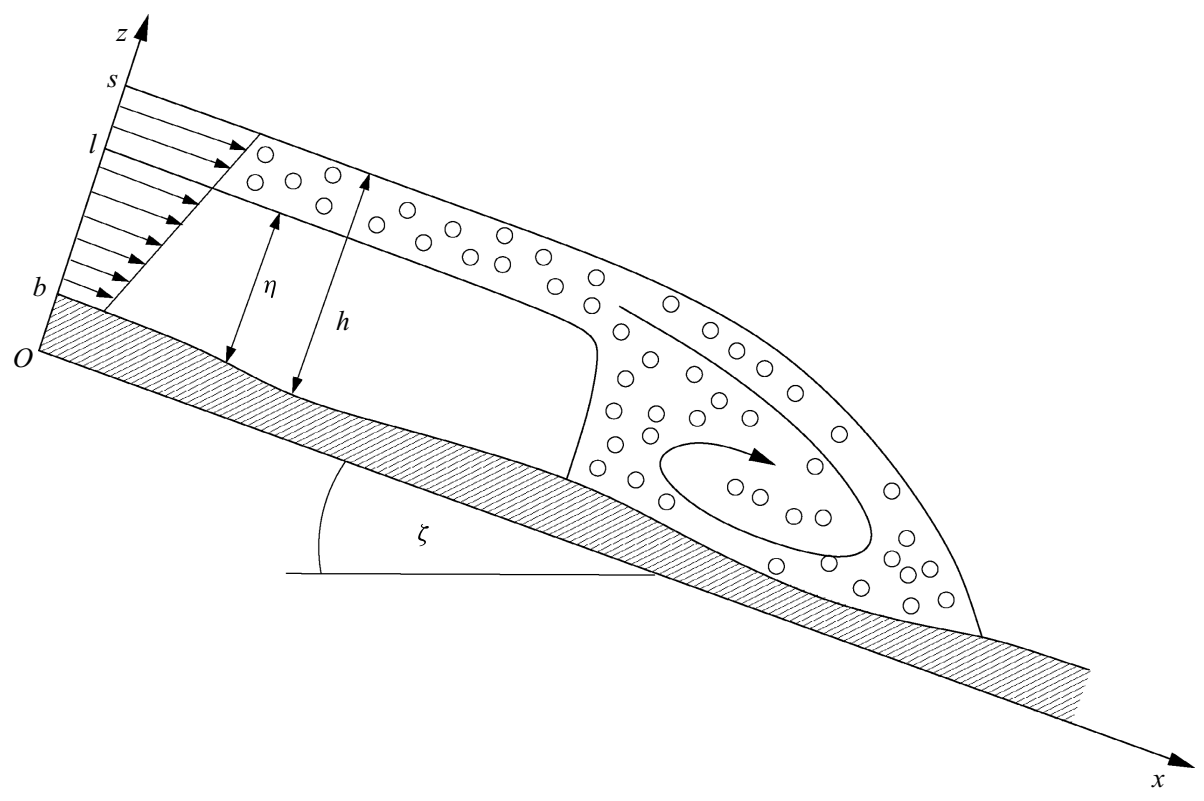

FIGURE 1. A schematic diagram of an avalanche flow front and the inversely graded particle size distribution within the interior. Large particles rise up into the faster-moving near-surface layers of the avalanche and are transported to the flow front, where they can be overrun and then recirculated, by particle size segregation, to form a bouldery margin. The basal layer of small particles is of thickness $\eta$ and the total depth of the avalanche is $h$. The downslope coordinate $x$ is inclined at an angle $\zeta$ to the horizontal.

Lun 1988). As the grains are sheared past one another, the avalanche dilates sufficiently for small particles to be able to preferentially percolate down into gaps that open up beneath them under gravity and, once underneath, they gradually lever the larger particles upwards to equilibrate the solids volume fraction and pressure. Larger particles therefore tend to rise towards the surface of the flow and the smaller particles percolate down to the bottom to create an inversely graded' particle size distribution (Middleton \& Hampton 1976). Since the upper layers of the avalanche move faster than the lower ones, there is a net transport of large particles towards the flow front as shown in the schematic diagram in figure 1. Once the coarse grains reach the front they may accumulate, either by being overrun and recirculated again by particle size segregation (Costa \& Williams 1984; Pierson 1986; Iverson 1997; Pouliquen, Delour \& Savage 1997) or by being pushed along en masse in front of the flow (Pouliquen \& Vallance 1999).

The accumulation of larger less mobile particles at avalanche fronts can have a significant influence on the bulk motion of geophysical mass flows, and is the source of instabilities that can spontaneously generate self-channelizing leveed flows (Pierson 1986; Iverson \& Vallance 2001; Iverson 2003) and digitate lobate terminations (Calder, Sparks \& Gardeweg 2000). The photograph in figure 2 shows an example of this in a snow avalanche that has uprooted trees and entrained debris as it destroyed approximately $200 \mathrm{~m}^{2}$ of forest in the Puschlav valley. The deposit has a welldeveloped snout that is almost entirely composed of tree stumps and coarse debris, and the interior is bounded by lateral levees that are composed of the same material as the front. Such deposits are formed by the larger more resistive material that reaches the front, being continuously shouldered aside by the more mobile interior, to create 


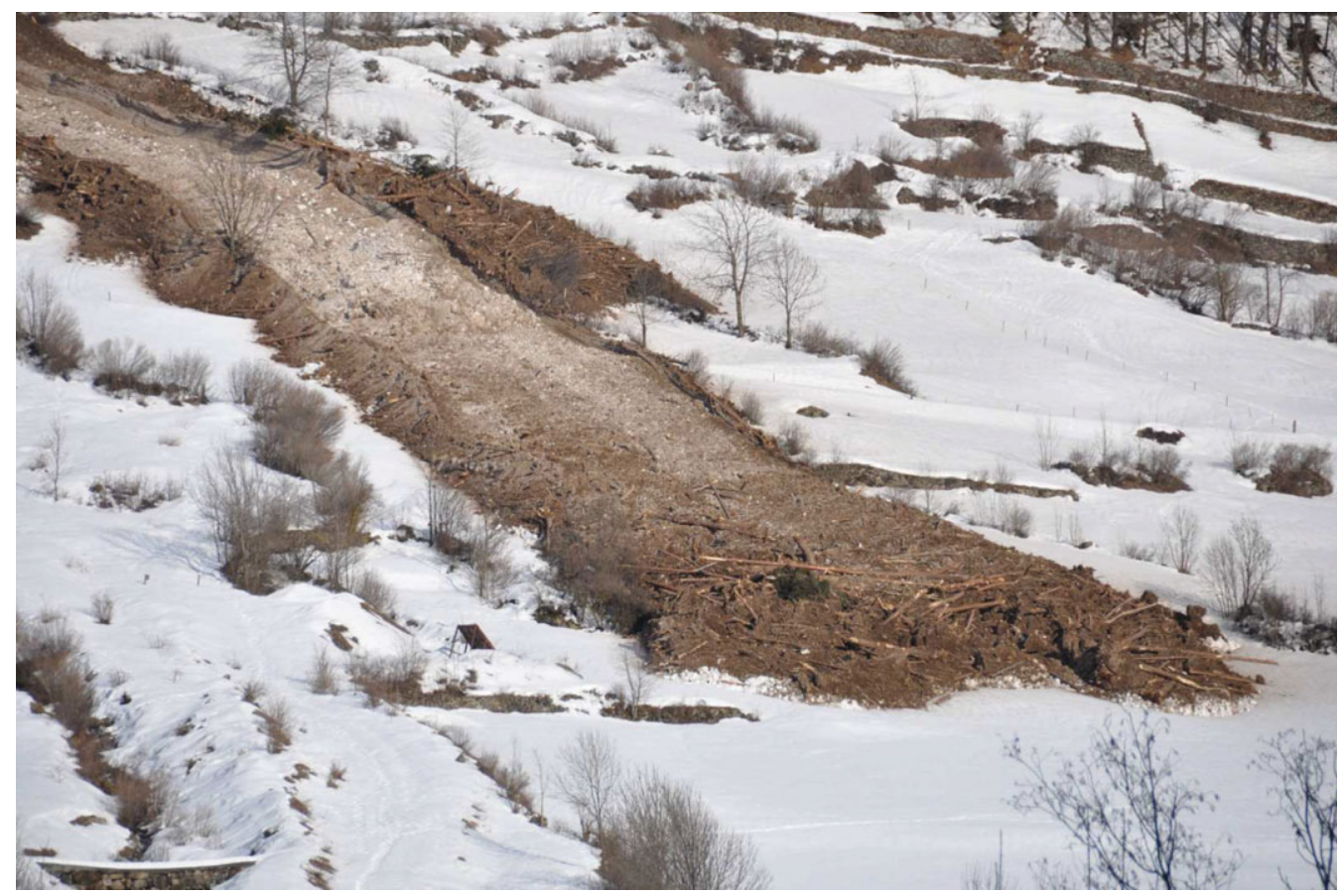

Figure 2. Snow avalanche with levees formed from debris at Val Prada, Switzerland (Courtesy P. Bartellt, WSL-Institut für Schnee- und Lawinenforschung, Davos). The avalanche occurred on the Val Prada torrent, located near Poschiavo in the Puschlav valley on the 9th February 2009. The avalanche was released from five different zones in a large basin and was estimated to be between 100000 to $150000 \mathrm{~m}^{3}$. One of the zones was located above a forest, and approximately $200 \mathrm{~m}^{2}$ was destroyed, entraining dirt, trees and scree into the avalanche. In this picture the slope is about $10^{\circ}$ and eyewitnesses report the avalanche travelling at constant velocity of $2-5 \mathrm{~m} \mathrm{~s}^{-1}$ before coming to rest.

stationary lateral levees that channelize the flow and enhance the overall run-out distance. Similar features are also observed in both wet and dry snow avalanches (e.g. Jomelli \& Bertran 2001; Bartelt \& McArdell 2009), water-saturated debris flows (e.g. Pierson 1986; Iverson 1997, 2003), rock avalanches (e.g. Bertran 2003) and dense pyroclastic flows (e.g. Calder et al. 2000; Iverson \& Vallance 2001). On a much smaller laboratory scale, fingering instabilities and leveed channels can also be produced with dry mixtures of large rough grains and smaller smoother particles (e.g. Pouliquen et al. 1997; Pouliquen \& Vallance 1999; Félix \& Thomas 2004; Aranson, Malloggi \& Clement 2006; Goujon, Dalloz-Dubrujeaud \& Thomas 2007).

All of these flows are examples of 'segregation-mobility feedback' phenomena in which the evolving particle size distribution has a direct effect on the local mobility of the avalanche. Other examples include longer run-out caused by segregation-induced changes to the velocity profile (Phillips et al. 2006; Linares-Guerrero, Goujon \& Zenit 2007; Rognon et al. 2007), petal formation in rotating drums (Hill, Gioia \& Amaravadi 2004; Zuriguel et al. 2006) and the formation of two-dimensional stratification patterns in Hele-Shaw cells (Williams 1968; Grasselli \& Herrmann 1997; Gray \& Hutter 1997; Makse et al. 1997; Baxter et al. 1998), where the more resistive grains that accumulate at the flow front are deposited onto the underlying substrate, rather than being continuously shouldered aside into lateral levees (Gray \& Ancey 2009). 
In order to properly understand some of these important and complex effects, it is necessary to develop mathematical models that can not only compute the evolving particle size distribution within the avalanche, but the effect that this has on (i) the local mobility of the flow, (ii) the velocity profile through the depth of the avalanche and (iii) the basal deposition. One of the major obstacles in achieving this goal is that nearly all existing models for granular avalanches use a system of depth-averaged equations to compute the thickness $h$ and depth-averaged velocity $\overline{\boldsymbol{u}}$ (e.g. Grigorian, Eglit \& Iakimov 1967; Savage \& Hutter 1989; Iverson 1997; Gray, Wieland \& Hutter 1999; Pouliquen 1999a; Wieland, Gray \& Hutter 1999; Iverson \& Denlinger 2001; Gray, Tai \& Noelle 2003; Pitman et al. 2003; Gruber \& Bartelt 2007; Mangeney et al. 2007). Whereas simple theories for kinetic sieving and squeeze expulsion require the full three-dimensional velocity field $\boldsymbol{u}$ (Savage \& Lun 1988; Dolgunin \& Ukolov 1995; Gray \& Thornton 2005; Gray \& Chugunov 2006; Thornton, Gray \& Hogg 2006). It is possible to reconstruct $\boldsymbol{u}$ from $\overline{\boldsymbol{u}}$ using bulk incompressibility and assumed velocity profiles through the avalanche depth (Gray \& Ancey 2009) to solve for the three-dimensional concentration of small particles $\phi$. It would, however, be very useful to have a description that fitted naturally into the depth-averaged avalanche framework. This paper addresses this need by deriving a new equation that is able to capture the essential processes involved in the transport of large particles towards a flow front.

\section{Governing equations}

In this section the depth-averaged avalanche equations and the particle size segregation equations are briefly reviewed, before using some of the results to derive a new depth-averaged theory for large particle transport in granular avalanches.

\subsection{Depth-averaged avalanche models}

Let $O x z$ define a coordinate system with the $x$ axis pointing down a slope inclined at an angle $\zeta$ to the horizontal and the $z$ axis being the upward pointing normal as shown in figure 1 . The avalanche velocity $\boldsymbol{u}$ has components $u(x, z, t)$ and $w(x, z, t)$ in the downslope and normal directions, respectively. The free surface of the avalanche lies at a height $s(x, t)$ and the basal topography on which it flows is defined by its height $b(x)$. The difference defines the avalanche thickness

$$
h(x, t)=s(x, t)-b(x) .
$$

Although there is some dilation and contraction of the material, it is commonly assumed that the avalanche is incompressible

$$
\frac{\partial u}{\partial x}+\frac{\partial w}{\partial z}=0
$$

which is a reasonable first approximation. This can be integrated through the avalanche depth by using Leibniz' integral theorem (e.g. Abramowitz \& Stegun 1970) for exchanging the order of integration and differentiation with respect to a variable $\lambda$

to give

$$
\frac{\partial}{\partial \lambda} \int_{b(\lambda)}^{s(\lambda)} f \mathrm{~d} z=\int_{b(\lambda)}^{s(\lambda)} \frac{\partial f}{\partial \lambda} \mathrm{d} z+\left[f \frac{\partial z}{\partial \lambda}\right]_{b(\lambda)}^{s(\lambda)},
$$

$$
\int_{b}^{s}\left(\frac{\partial u}{\partial x}+\frac{\partial w}{\partial z}\right) \mathrm{d} z=\frac{\partial}{\partial x}\left(\int_{s}^{b} u \mathrm{~d} z\right)-\left[u \frac{\partial z}{\partial x}-w\right]_{b}^{s}
$$


Defining the depth-averaged avalanche velocity

$$
\bar{u}=\frac{1}{h} \int_{b}^{s} u \mathrm{~d} z
$$

and using the kinematic boundary conditions (e.g. Savage \& Hutter 1989)

$$
\frac{\partial z}{\partial t}+u \frac{\partial z}{\partial x}-w=0, \quad \text { at } \quad z=s(x, t) \quad \text { and } \quad z=b(x)
$$

the depth-averaged mass balance becomes

$$
\frac{\partial h}{\partial t}+\frac{\partial}{\partial x}(h \bar{u})=0 .
$$

A similar integration procedure that incorporates the surface and basal kinematic and traction boundary conditions can be used to derive a depth-integrated momentum balance equation in the downslope direction (e.g. Savage \& Hutter 1989; Gray et al. 1999, 2003). Assuming that the avalanche is shallow, the normal momentum balance implies that the pressure is lithostatic

$$
p=\rho g(s-z) \cos \zeta
$$

where $\rho$ is the density of the material and $g$ is the constant of gravitational acceleration. To close the model it is necessary to include a simplified representation of the normal stress gradients in the downslope direction. There are essentially two variants of the theory; one group that uses a simplified Mohr-Coulomb rheology (e.g. Savage \& Hutter 1989; Iverson 1997; Gray et al. 1999; Iverson \& Denlinger 2001) and another that assumes that the internal stress is dominated by the isotropic pressure field (e.g. Grigorian et al. 1967; Pouliquen 1999a; Gray et al. 2003; Pitman et al. 2003; Cui, Gray \& Johannesson 2007; Gray \& Cui 2007; Gruber \& Bartelt 2007; Mangeney et al. 2007). In both cases the resulting depth-averaged downslope momentum balance takes the form

$$
\frac{\partial}{\partial t}(h \bar{u})+\frac{\partial}{\partial x}\left(F h \bar{u}^{2}\right)+\frac{\partial}{\partial x}\left(\frac{1}{2} K g \cos \zeta h^{2}\right)=h g \cos \zeta\left(\tan \zeta-\frac{u}{|u|} \mu-\frac{\partial b}{\partial x}\right),
$$

where $F=\overline{u^{2}} / \bar{u}^{2}$ is the velocity shape factor and $\mu$ is the coefficient of basal friction. For the Mohr-Coulomb models, the earth pressure coefficient $K$ jumps between two limiting stress states as the avalanche switches from divergence to convergence (see e.g. Savage \& Hutter 1989). In the isotropic pressure models $K=1$ and (2.7) and (2.9) reduce to the same mathematical structure as the shallow water equations. The source term on the right-hand side of (2.9) represents the physical effects of gravitational acceleration, basal friction and basal topography gradients.

\subsection{Models for particle size segregation and diffusive remixing}

Savage \& Lun (1988) derived the first theory for segregation in granular avalanches using information entropy ideas, and Dolgunin \& Ukolov (1995) wrote down a similar model that included the diffusive effects of particle remixing. More recently Gray \& Thornton (2005), Thornton et al. (2006) and Gray \& Chugunov (2006) have derived segregation models from mixture theory, which are compatible with the assumptions made in the derivation of the depth-averaged avalanche equations, i.e. the flow is shallow, it satisfies bulk incompressibility (2.2) and has a lithostatic pressure distribution through its depth (2.8). The processes of kinetic sieving and squeeze expulsion are modelled as a percolation process that is driven by perturbations to 
the lithostatic pressure distribution. The fines carry less of the load as they percolate downwards and the large grains support correspondingly more of the overburden pressure, until they separate out into a pure phase. This places restrictions on the functional form of the segregation flux (Gray \& Thornton 2005), which is presented here in its simplest form. While the derivations of Gray and co-workers differ from that of Savage \& Lun (1988) and Dolgunin \& Ukolov (1995), all of these theories take the general form

$$
\frac{\partial \phi}{\partial t}+\frac{\partial}{\partial x}(\phi u)+\frac{\partial}{\partial z}(\phi w)-\frac{\partial}{\partial z}(q \phi(1-\phi))=\frac{\partial}{\partial z}\left(D \frac{\partial \phi}{\partial z}\right),
$$

where $0 \leqslant \phi \leqslant 1$ is the volume fraction of small particles per unit granular volume, $q$ is the mean segregation velocity, $D$ is the coefficient of diffusive remixing and the volume fraction of large particles is equal to $1-\phi$. The segregation flux, $-q \phi(1-\phi)$, has the property that it shuts off when there are either all large particles or all fines, which automatically keeps $\phi \in[0,1]$. The segregation rate $q$ and the diffusion coefficient $D$ may contain further dependencies on $\phi$ (e.g. Savage \& Lun 1988), as well as the grain size ratio, the shear rate and the local solids volume fraction, but, so far, there has been very little experimental work (e.g. Savage \& Lun 1988; Valance \& Savage 2000; Golick \& Daniels 2009) or discrete element simulations (e.g. Khakhar, McCarthy \& Ottino 1999; Gray \& Chugunov 2006) to determine these functional dependencies.

Equation (2.10) is subject to the condition that there is no flux of small particles across the surface and basal boundaries

$$
q \phi(1-\phi)+D \frac{\partial \phi}{\partial z}=0 \quad \text { at } \quad z=s(x, t), \quad \text { and } \quad z=b(x) .
$$

The segregation-remixing equation (2.10) is defined in two space dimensions and requires the two-dimensional velocity components $(u, w)$ of the bulk flow field. These can be reconstructed from $\bar{u}$ using assumed downslope velocity profiles through the avalanche depth, together with the incompressibility condition (2.2), as in Gray \& Ancey (2009). Alternatively $(u, w)$ can be computed directly using the new constitutive law for dense granular avalanches (Jop, Forterre \& Pouliquen 2006), which Rognon et al. (2007) have suggested may be modified for binary mixtures. A third possibility is to incorporate a simplified description of the segregation process into a depthaveraged model, i.e. to reduce the dimension of the segregation equation, so that there is no need to reconstruct the velocity components $(u, w)$.

\subsection{Derivation of a depth-averaged segregation model}

Defining the depth-averaged concentration of small particles and the depth-averaged flux of small particles as

$$
\bar{\phi}=\frac{1}{h} \int_{b}^{s} \phi \mathrm{d} z, \quad \text { and } \quad \overline{\phi u}=\frac{1}{h} \int_{b}^{s} \phi u \mathrm{~d} z
$$

the segregation-remixing equation (2.10) can be integrated through the avalanche depth using Leibniz' integral theorem (2.3) to give

$$
\frac{\partial}{\partial t}(h \bar{\phi})+\frac{\partial}{\partial x}(h \bar{\phi} \bar{u})-\left[\phi\left(\frac{\partial z}{\partial t}+u \frac{\partial z}{\partial x}-w\right)\right]_{b}^{s}=\left[q \phi(1-\phi)+D \frac{\partial \phi}{\partial z}\right]_{b}^{s} .
$$

The first square-bracketed term is identically zero due to the surface and basal kinematic conditions (2.6) and the second square-bracketed term is identically zero 
because of the no-flux condition (2.11). It follows that the depth-integrated segregation equation (2.13) reduces to

$$
\frac{\partial}{\partial t}(h \bar{\phi})+\frac{\partial}{\partial x}(h \overline{\phi u})=0
$$

which no longer has any explicit dependence on $q$ and $D$, although the depth-averaged concentration of small particles $\bar{\phi}$ and the depth-averaged small particle flux $\overline{\phi u}$ may still be dependent on both of these parameters.

In order to close, the model expressions must be devised for the depth-averaged quantities $\bar{\phi}$ and $\overline{\phi u}$ defined in (2.12). There are many possible model assumptions that could be made at this point, but we are motivated by the stratification pattern experiments of Gray \& Hutter (1997) and Gray \& Ancey (2009), which showed (i) that the inverse grading could develop very rapidly, (ii) that the grains could be very sharply segregated by size and (iii) that there was considerable evidence of strong shear through the avalanche depth. We will therefore assume that the avalanche segregates instantaneously, so that the particle size distribution is always sharply inversely graded, i.e.

$$
\phi= \begin{cases}0 & l \leqslant z \leqslant s \\ 1 & b \leqslant z \leqslant l\end{cases}
$$

This is equivalent to the assumption that $q \longrightarrow \infty$, which is a valid limit if the lateral length scale for complete segregation is much smaller than the typical length scale of the avalanche. The depth-averaged concentration is then equal to

$$
h \bar{\phi}=l-b=\eta,
$$

where $\eta$ is the thickness of the basal layer of small particles shown schematically in figure 1. Following Gray \& Thornton (2005) the downslope velocity is assumed to be linear with depth

$$
u=\alpha \bar{u}+2(1-\alpha) \bar{u}\left(\frac{z-b}{h}\right), \quad 0 \leqslant \alpha \leqslant 1,
$$

with a parameter $\alpha$ that allows the profile to vary from plug flow for $\alpha=1$, to simple shear for $\alpha=0$, and linear shear with basal slip for intermediate values. This is able to capture the leading-order behaviour of flows on both smooth (Savage \& Hutter 1989) and rough slopes (Gray \& Ancey 2009), although more general profiles are possible. Using (2.15) and (2.17) it follows that the depth-averaged flux of small particles

$$
h \overline{\phi u}=\eta \bar{u}-(1-\alpha) \bar{u} \eta\left(1-\frac{\eta}{h}\right),
$$

which is independent of the parameters $q$ and $D$ used in the segregation-remixing equation (2.10). Substituting (2.16) and (2.18) into (2.14) yields an important new equation for the evolution of the inversely graded shock interface height $\eta$

$$
\frac{\partial \eta}{\partial t}+\frac{\partial}{\partial x}(\eta \bar{u})-\frac{\partial}{\partial x}\left((1-\alpha) \bar{u} \eta\left(1-\frac{\eta}{h}\right)\right)=0 .
$$

An equivalent and almost identical equation for the evolution of the large particle thickness $\chi=h-\eta$ is obtained by subtracting (2.19) from (2.7) to give

$$
\frac{\partial \chi}{\partial t}+\frac{\partial}{\partial x}(\chi \bar{u})+\frac{\partial}{\partial x}\left((1-\alpha) \bar{u} \chi\left(1-\frac{\chi}{h}\right)\right)=0 .
$$


In general, either (2.19) or (2.20) can be used to calculate the inversely graded interface position. In this paper we will use (2.19). We will refer to it as the 'large particle transport equation' or 'transport equation' for short, because it preferentially transports large particles towards the avalanche front. The easy way to see this is to calculate the large and small particle transport velocities from (2.20) and (2.19), which are

$$
\begin{aligned}
\bar{u}^{l} & =\bar{u}+(1-\alpha) \bar{u} \eta / h, \\
\bar{u}^{s} & =\bar{u}-(1-\alpha) \bar{u} \chi / h,
\end{aligned}
$$

respectively. The depth-averaged velocity $\bar{u}$ determines the flow direction and hence the direction of the front. Equation (2.21) implies that provided there is shear, the large particles will move towards the front faster than the average velocity $\bar{u}$, unless there are no small particles in that region of the flow. While (2.22) implies that the small particles will be transported towards the flow front slower than average, unless there are no large particles left. The transport term in (2.19) has the property that it shuts off when $\eta=0, h$ and when the transport rate $(1-\alpha) \bar{u} / h$ is equal to zero. Since the transport rate is proportional to the downslope velocity shear through the avalanche depth, it is equal to zero when the material is stationary $(\bar{u}=0)$ and when there is plug flow $(\alpha=1)$. In this case the transport equation (2.19) degenerates to the same form as the thickness equation (2.7) and $h / \eta$ is conserved.

The large particle transport equation represents the first attempt to include size segregation and shear into the depth-averaged avalanche framework. It encapsulates the key physical effects that drive the transport of large grains towards a flow front. Despite its simplicity (2.19) has considerable power because it reduces a twodimensional problem to a one-dimensional one. This opens up the realistic possibility of coupling the basal friction $\mu$ of the bulk flow to the evolving particle size distribution within the avalanche, to model the subtle 'segregation-mobility' feedback effects discussed by Pouliquen et al. (1997), Pouliquen \& Vallance (1999) Iverson \& Vallance (2001), Phillips et al. (2006) and Gray \& Ancey (2009). The properties of the transport equation (2.19) will be investigated in this paper, using either prescribed avalanche thickness and velocity distributions or simplified uncoupled solutions for the bulk flow.

\subsection{Non-dimensionalization}

It is convenient to introduce non-dimensional variables that reflect the shallowness of the avalanche and typical magnitudes for the thickness $H$, length $L$ and velocity $U$

$$
x=L \tilde{x}, \quad(z, h, \eta)=H(\tilde{z}, \tilde{h}, \tilde{\eta}), \quad t=(L / U) \tilde{t}, \quad(u, \bar{u}, w)=U(\tilde{u}, \tilde{\bar{u}}, \varepsilon \tilde{w}),
$$

where the aspect ratio of the avalanche $\varepsilon=H / L$ is small. In the conventional scaling for smooth beds (Savage \& Hutter 1989), the velocity magnitude $U=\sqrt{L g}$ is set by a balance between acceleration and gravity. For rough beds a different velocity magnitude arises (Gray \& Ancey 2009), because the gravitational acceleration is balanced by the frictional resistance to motion (Pouliquen 1999b). Since we are investigating uncoupled solutions to the transport equation in this paper, the magnitude of $U$ is left as general as possible at this stage. With the scalings (2.23), the non-dimensional form of the large particle transport equation (2.19) remains essentially unchanged

$$
\frac{\partial \eta}{\partial t}+\frac{\partial}{\partial x}(\eta \bar{u})-\frac{\partial}{\partial x}\left((1-\alpha) \bar{u} \eta\left(1-\frac{\eta}{h}\right)\right)=0,
$$


where the tildes are dropped for simplicity. Using the identity

$$
\bar{\phi}=\frac{\eta}{h},
$$

the transport equation (2.24) can be rewritten as an equation for the depth-averaged concentration of small particles

$$
\frac{\partial}{\partial t}(h \bar{\phi})+\frac{\partial}{\partial x}(h \bar{\phi} \bar{u})-\frac{\partial}{\partial x}((1-\alpha) h \bar{u} \bar{\phi}(1-\bar{\phi}))=0 .
$$

This is very closely analogous to the non-dimensional segregation equation without diffusive effects $(D=0)$, which becomes

$$
\frac{\partial \phi}{\partial t}+\frac{\partial}{\partial x}(\phi u)+\frac{\partial}{\partial z}(\phi w)-\frac{\partial}{\partial z}\left(S_{r} \phi(1-\phi)\right)=0,
$$

where $S_{r}=q L /(H U)$ is the non-dimensional segregation number. Equation (2.26) is one-dimensional and has a quadratic dependence on $\bar{\phi}$ in the $x$ direction, while (2.27) is two-dimensional and has a quadratic dependence on $\phi$ in the $z$ direction. Previous analysis of the hyperbolic segregation equation (e.g. Gray \& Thornton 2005; Gray, Shearer \& Thornton 2006; Thornton et al. 2006; Shearer, Gray \& Thornton 2008) implies that small particles tend to percolate downwards with speed $w^{s}=w-S_{r}(1-\phi)$, while large particles tend to rise with velocity $w^{l}=w+S_{r} \phi$, collecting in a pure phase adjacent to the free surface. Since the segregation velocities are almost identical in form to the transport velocities (2.22) and (2.21) in the depth-averaged model, this immediately suggests that the transport equation (2.24) will develop higher concentrations of large particles near the avalanche front.

\section{Interface transport and breaking size segregation waves}

The transport equation (2.24) assumes that the segregation rate is infinite and that the large particles are always inversely graded. While this achieves great simplification, it means the theory can not represent the detailed structure of the two-dimensional expansion fans and concentration shocks (Gray \& Thornton 2005; Gray et al. 2006; Thornton et al. 2006; Shearer et al. 2008; Gray \& Ancey 2009) that enable the particles to readjust into a stable inversely graded configuration. The theory, however, does a remarkably good job of representing solutions to problems once the grains have become inversely graded as will be shown here.

\subsection{Interface transport}

Consider the transport of a monotonically decreasing shock interface $\eta$. Assuming that the avalanche is steady and uniform, the large particle transport equation (2.24) becomes

$$
\frac{\partial \eta}{\partial t}+\frac{\partial}{\partial x}\left(\alpha \eta+(1-\alpha) \eta^{2}\right)=0,
$$

where $\bar{u}$ and $h$ are assumed to be equal to unity by virtue of the scalings (2.23). Expanding out the derivatives this can also be written as

$$
\frac{\partial \eta}{\partial t}+u(\eta) \frac{\partial \eta}{\partial x}=0
$$

where the non-dimensional downslope velocity

$$
u(\eta)=\alpha+2(1-\alpha) \eta .
$$


Equation (3.2) has exactly the same form as that derived by Gray et al. (2006) for the evolution of the shock separating large particles above from small particles below (see their equation (4.20)), and closely resembles the inviscid Burgers' equation. Hence, provided that the particles are already sharply segregated and the shock interface does not break, the depth-averaged model (2.24) and the full segregation theory (2.26) give precisely the same result.

The position of the shock interface can be computed by the method of characteristics. For initial data $\left(x_{o}, \eta_{o}\right)$ at $t=t_{o}$ the solution is

$$
\eta=\eta_{o}, \quad \text { on } \quad x=x_{o}+u\left(\eta_{o}\right)\left(t-t_{o}\right) .
$$

An example of this is shown in figure 3 for an initial discontinuity at $x=0$ between constant upstream height $\eta_{u p}=0.1$ and downstream height $\eta_{\text {down }}=0.9$. Since the velocity is greater nearer the top of the flow the downstream interface moves faster than the upstream side and an expansion fan forms. The interface height within this expansion is linear in $x$ and its gradient decreases with increasing time

$$
\eta=\frac{1}{2(1-\alpha)}\left(\frac{x}{t}-\alpha\right), \quad x \in\left[u\left(\eta_{u p}\right) t, u\left(\eta_{\text {down }}\right) t\right] .
$$

The expansion fan is centred at $x=0$ at $t=0$ and can be seen more clearly in terms of characteristics on the $(x, t)$ diagram in figure 4 . Characteristic lines with the lower angle of inclination correspond to interfaces that are higher in the flow and move faster downstream.

\subsection{Breaking size segregation waves}

Breaking size segregation waves (Thornton \& Gray 2008; Gray \& Ancey 2009) are a very important and common feature of bi-disperse granular flows and form whenever small grains are sheared over the top of large particles. Within these waves small particles percolate down into the slower moving layers of the avalanche and squeeze the larger ones up into the faster moving flow near the free surface. Breaking size segregation waves therefore recirculate large particles back towards the front of an avalanche and the smaller ones towards the tail. A simple compression problem with a monotonic decrease in the interface height $\eta$ is sufficient to generate a breaking wave at some finite time later. For instance, Thornton \& Gray (2008) assumed that the interface

$$
\eta= \begin{cases}0.9, & -0.8>x, \\ (1-x) / 2, & -0.8 \leqslant x \leqslant 0.8, \\ 0.1, & 0.8<x,\end{cases}
$$

as shown in figure 5. Prior to the wave breaking the steepening of the interface is the same for both the depth-averaged transport theory and the two-dimensional segregation model. The characteristics (3.4) and the initial condition (3.6) imply that for $\alpha=0$ the linear section of the interface is given by

$$
\eta=\frac{x-1}{2(t-1)}, \quad-0.8+1.8 t \leqslant x \leqslant 0.8+0.2 t,
$$

and it becomes vertical at $x=1$ at time $t=1$. After $t=1$ a breaking wave forms in the two-dimensional size segregation theory (Thornton \& Gray 2008), which propagates downstream at approximately unit speed and oscillates backwards and forwards like a spinning rugby ball as shown in figure 5. The structure of the time-dependent breaking wave is complex and so far exact solutions have been generated only for the 

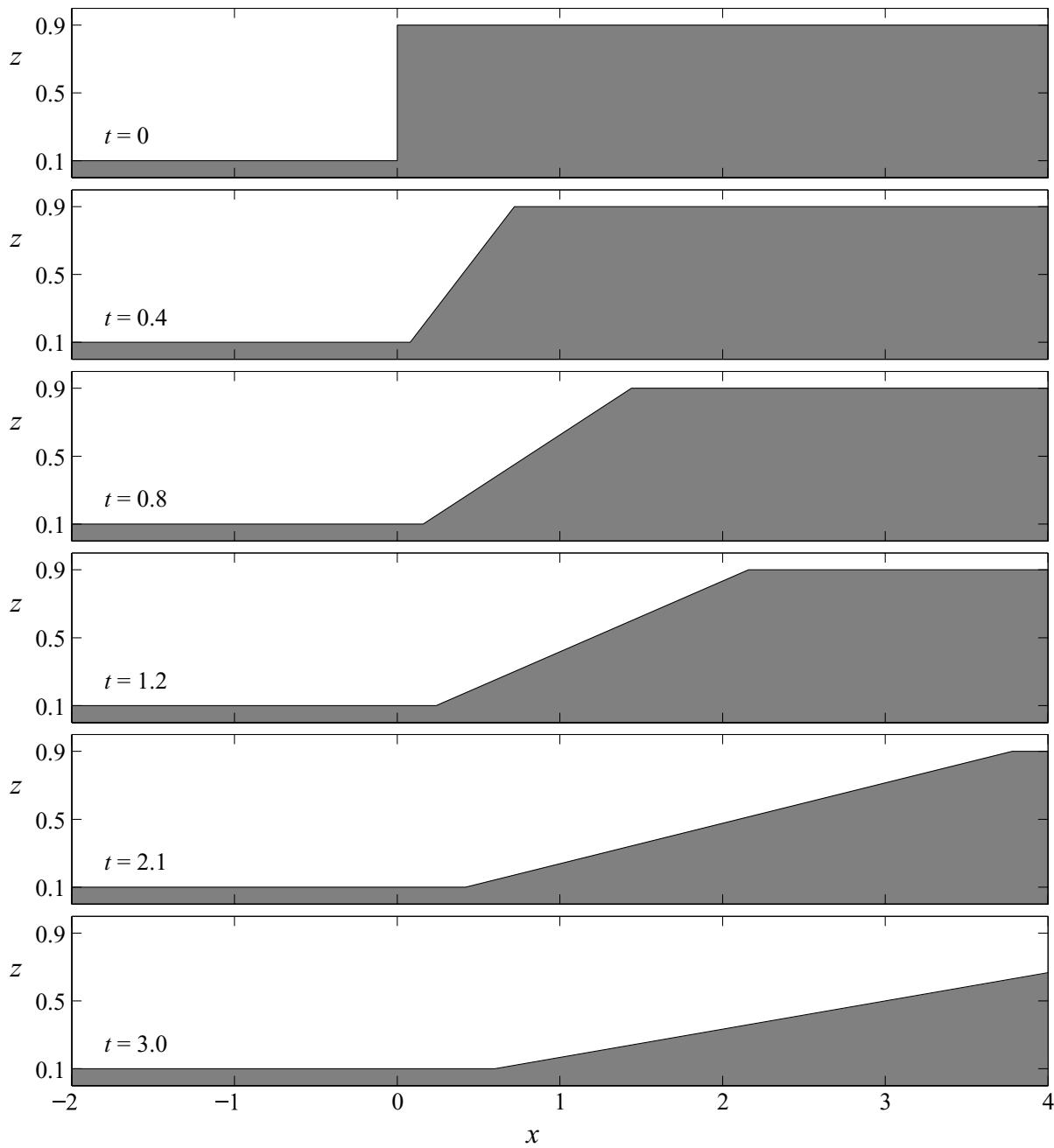

FiguRE 3. A series of vertical sections through a unit-thickness avalanche showing the evolution of the particle size distribution with increasing time. There is a linear downslope velocity profile through the avalanche depth with no slip at the base $(\alpha=0)$. The avalanche is sharply segregated with all the large particles (white) above the small (grey) ones. The shock interface $\eta$ between the two species has an initial discontinuity at $x=0$ and lies at a height 0.1 upstream and 0.9 downstream. At subsequent times this forms an expansion fan with a linear transition between the two states. The solution is identical for both the one-dimensional depth-averaged transport theory and the full two-dimensional hyperbolic segregation model.

initial phase of breaking (McIntyre et al. 2007). Numerical simulations (Thornton \& Gray 2008) indicate that the oscillations slowly decay to leave an exact steady-state solution (Thornton \& Gray 2008), which consists of two expansions fans and two shocks that are arranged in a lens-like structure. The numerical solution shown in figure 5 is computed using a non-oscillatory central scheme with a minmod slope limiter (Nessyahu \& Tadmor 1990; Jiang et al. 1998; Jiang \& Tadmor 1998), which is a high-resolution shock-capturing method that has successfully been used to compute the flow of avalanches past obstacles (Tai et al. 2002; Gray et al. 2003; Cui et al. 2007; Gray \& Cui 2007). 


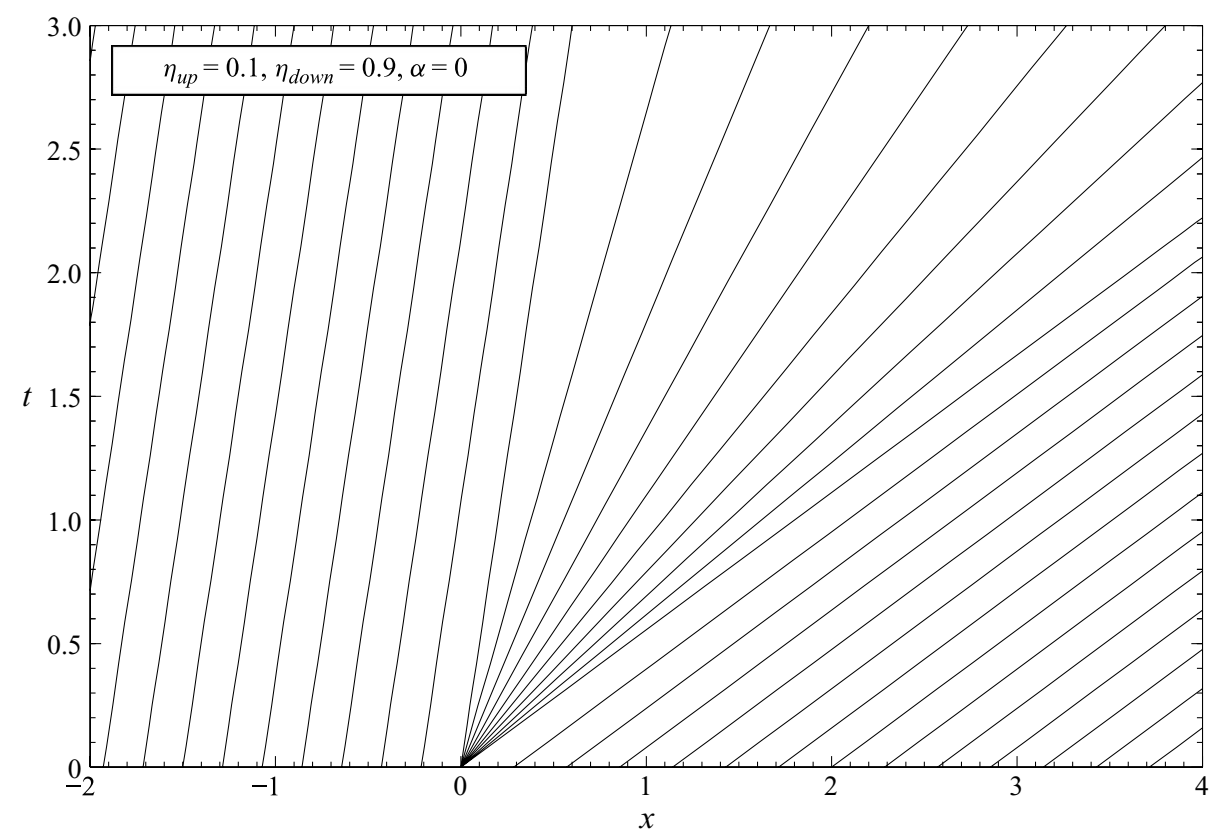

FIgURE 4. An $(x, t)$ diagram corresponding to the problem in figure 3 , showing the characteristic lines and the expansion fan centred at $x=0$ at $t=0$. The characteristics are more steeply inclined for lower interface heights $\eta$ and hence information propagates more slowly downstream.

In the equivalent large particle transport problem the breaking size segregation wave is replaced by a discontinuity in the shock interface height $\eta$. This is shown as a solid line in figure 5. At such discontinuities $\eta$ satisfies the jump condition (see e.g. Chadwick 1999; Gray et al. 2006, for a general derivation)

$$
\eta\left(\bar{u}-v_{n}\right)=\llbracket(1-\alpha) \bar{u} \eta\left(1-\frac{\eta}{h}\right) \rrbracket,
$$

where $v_{n}$ is the normal speed of the shock and the jump bracket $f=f_{2}-f_{1}$ is the difference of the enclosed quantity on the forward and rearward sides of the shock (denoted by the subscripts 2 and 1, respectively). For a unit-thickness avalanche with depth-averaged velocity $\bar{u}=1$ this implies that the shock speed

$$
v_{n}=\alpha+(1-\alpha)\left(\eta_{u p}+\eta_{\text {down }}\right),
$$

which is precisely the same as the speed of the steadily breaking wave found by Thornton \& Gray (2008) (see their equation (2.25)). For $\alpha=0$ the shock position

$$
x_{\text {shock }}=t, \quad t>1 \text {. }
$$

The complete structure of the characteristics and the shock in the depth-averaged solution is shown on an $(x, t)$ diagram in figure 6 . The characteristics in the linearly inclined section in (3.6) converge to the point $(1,1)$ where a shock is formed and then propagates downstream with unit speed. A comparison of the two-dimensional segregation theory and the one-dimensional transport solution shown in figure 5 shows that they are identical prior to $t=1$, and, at subsequent times, the complex structure of the breaking wave is replaced by a shock that travels at the correct speed. 


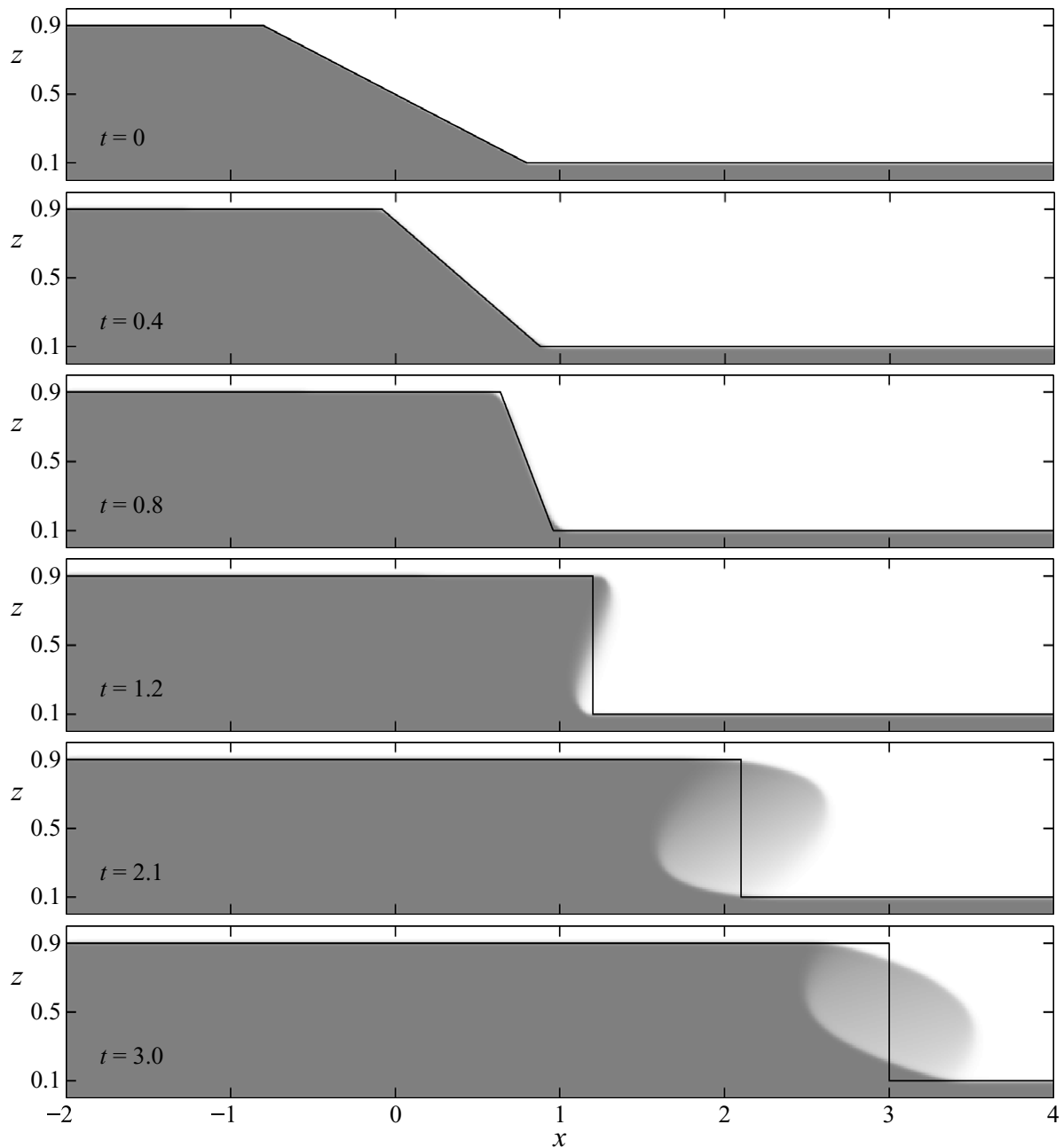

FiguRE 5. A comparison between the solution of the steepening wave problem of Thornton $\&$ Gray (2008) using the one-dimensional transport equation and that using the two-dimensional segregation equation. Initially the upstream interface lies at height $\eta_{u p}=0.9$ and is connected to the downstream interface at height $\eta_{\text {down }}=0.1$ by a linear transition in the region $-0.8<x<0.8$. The two-dimensional solution is represented with a contour scale with darker grey corresponding to higher concentrations of small particles and white to purely large particles. The interface position in the one-dimensional theory is represented by a solid line. Up until $t=1$ the solutions are identical, but after $t=1$ a breaking size segregation wave forms in the two-dimensional theory. This is represented as a shock in the interface height in the one-dimensional theory. An animated version is available with the online version of this paper (supplementary movie 1 available at journals.cambridge.org/flm).

This is a considerable simplification of the theory, while still retaining the key features of the relative transport of large and small particles in the downstream direction.

\subsection{Breaking wave interaction}

The simple transport equation is also able to capture the essence of the lens interaction problem of Thornton \& Gray (2008). In this problem the initial interface lies at a 


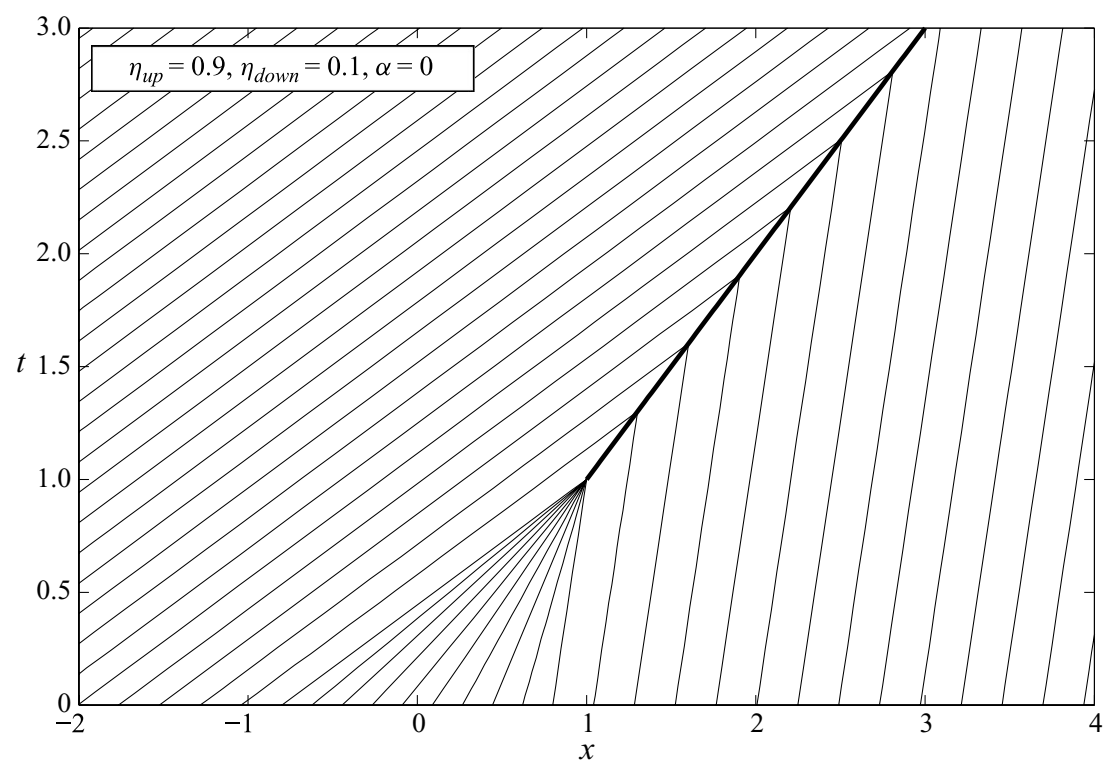

FIgURE 6. An $(x, t)$ diagram of the depth-averaged solution of the breaking wave problem of Thornton \& Gray (2008) showing the characteristics as thin lines and the shock as a thick line. The linear section steepens and breaks along its entire length at time $t=1$ at position $x=1$ forming a shock that travels downstream at unit speed.

height

$$
\eta= \begin{cases}0.9, & -1.3>x, \\ 0.5, & -1.3 \leqslant x \leqslant 1.3, \\ 0.1, & 1.3<x,\end{cases}
$$

and generates two breaking size segregation waves that move at different speeds downstream as shown in figure 7. The higher one is faster than the lower one, because the bulk velocity $u$ is greater near the free surface. Shortly after $t=3$ the two waves interact and merge with one another to form a single breaking wave that moves at approximately unit speed downstream. In the equivalent transport problem the shock relation (3.8) can again be used to calculate the motion of the shocks that replace the breaking size segregation waves. For $\alpha=0$, it follows that the upper shock moves with non-dimensional speed 1.4 and the lower one at speed 0.6, and their positions are

$$
x_{\text {upper }}=-1.3+1.4 t, \quad x_{\text {lower }}=1.3+0.6 t,
$$

respectively. They meet at $x=3.25$ at time $t=3.25$ and merge to form a single shock that moves with unit speed downstream with position

$$
x_{\text {merge }}=t, \quad t>3.25,
$$

as shown in figure 7 . The complete evolution can be summarized in the $(x, t)$ diagram shown in figure 8 , which shows how the characteristics run into either side of all three shocks. 


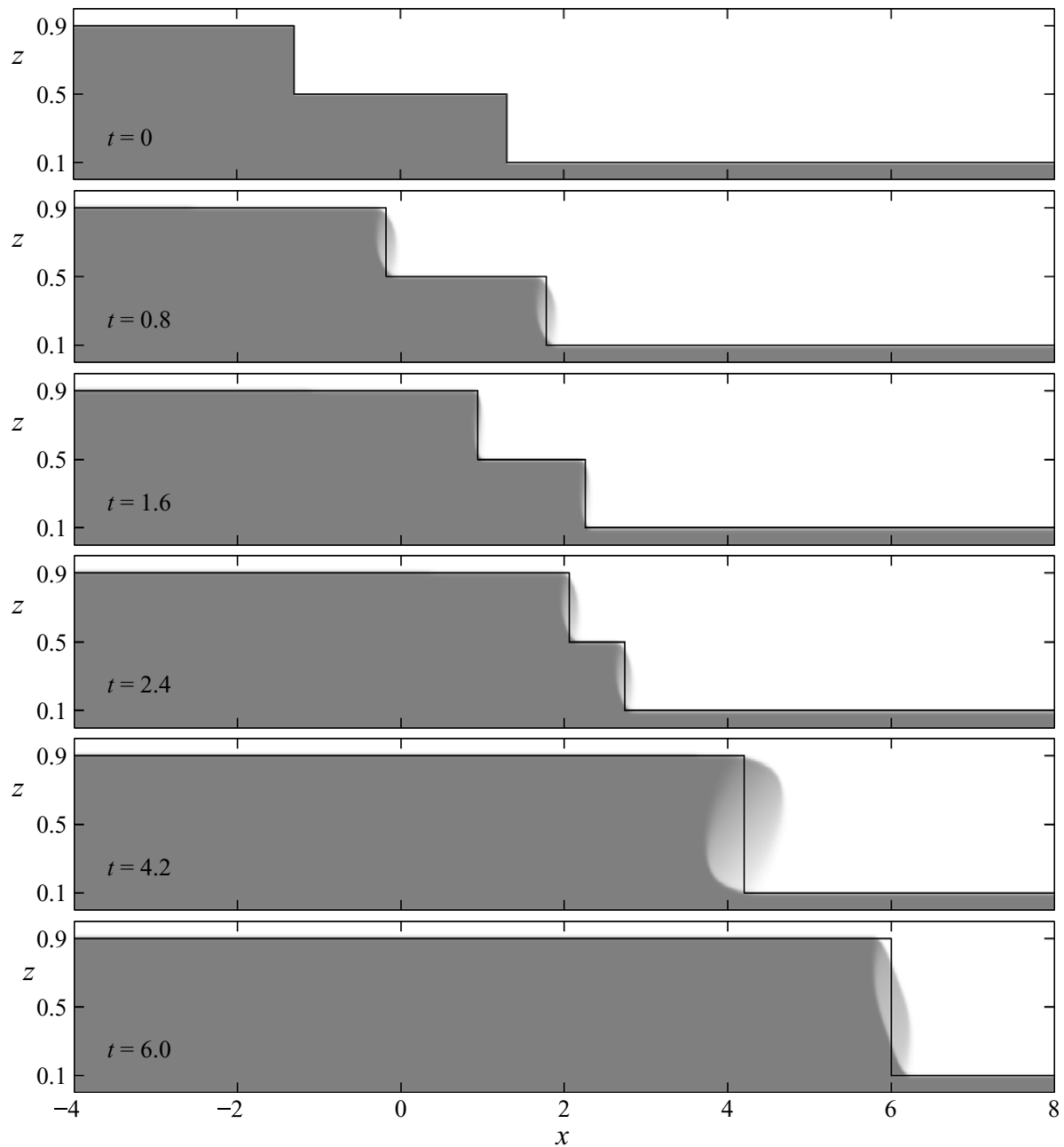

Figure 7. A comparison between the solution of the wave merging problem of Thornton \& Gray (2008) using the one-dimensional transport equation and that using the two-dimensional segregation equation. The same contour scale as in figure 5 is used. Initially the upstream interface has two discontinuities at $x=-1.3$ and 1.3 that separate an upstream section at height 0.9 from a middle section with height 0.5 and a downstream section of height 0.1 . The discontinuities form two breaking size segregation waves that propagate downstream and merge to form a single wave that moves at approximately unit speed. The one-dimensional transport solution (solid line) represents the breaking waves as shocks in the interface height. An animated version is available with the online version of this paper (supplementary movie 2).

\section{Stratification patterns}

Segregation often occurs when granular materials are poured into a heap for storage or filled into silos and bins (Williams 1968; Makse et al. 1997). This can be a significant problem in industry, where inconsistent blending degrades the quality and consistency of the product (Johanson 1978). Various types of patterns form in the deposits, which reflect both the roughness and size of the particles (Herrmann 1998) and the rate at which they are poured (Gray \& Hutter 1997; Baxter et al. 1998). At higher rates, when basal deposition is the dominant mechanism for stopping the 


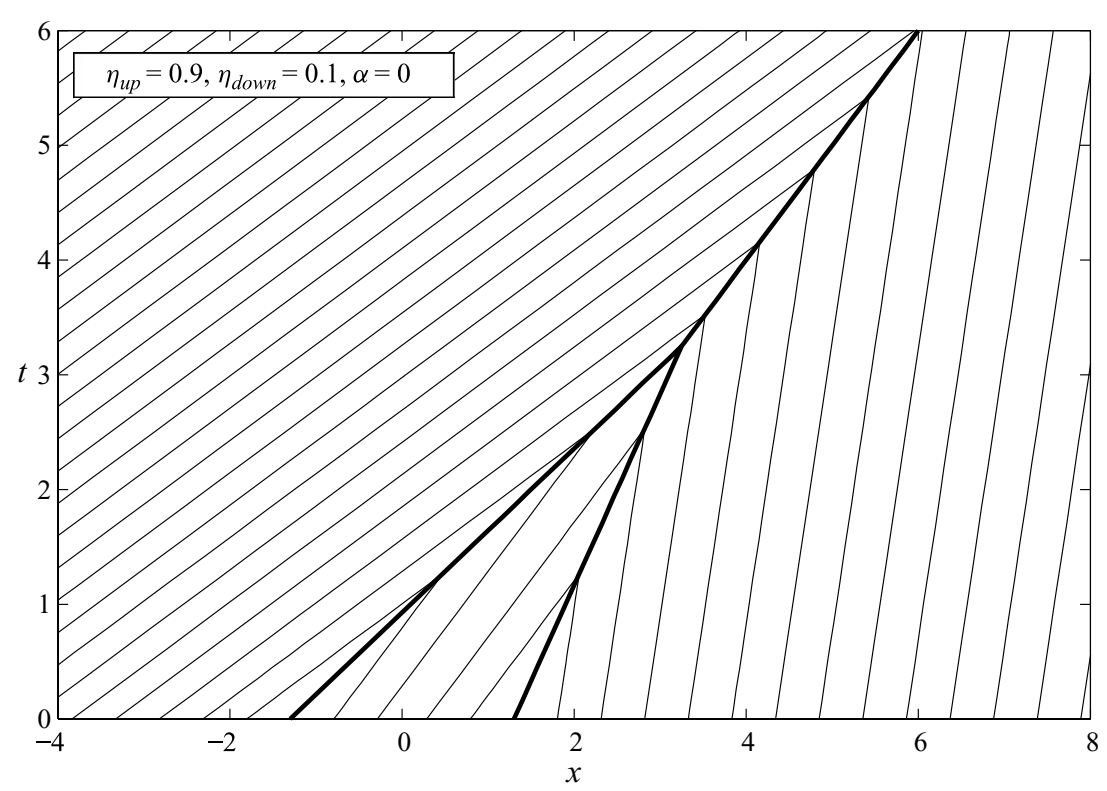

FIGURE 8. An $(x, t)$ diagram of the depth-averaged solution of the wave merging problem of Thornton \& Gray (2008) showing the characteristics as thin lines and the shocks as thick lines. The upper shock travels downstream faster than the lower one, and they merge at $t=3.25$ at $x=3.25$ to form a single shock that travels downstream at speed unity.

grains, a 'triangular' pattern forms with the highest concentrations of large particles farthest from the source. At lower flow rates, when the avalanche is brought to rest by the upslope propagation of a granular bore or shock wave (e.g. Gray \& Hutter 1997; Gray et al. 2003) the inversely graded layers in the avalanche are preserved in the deposit and form alternating stripes of large and small particles that lie parallel to the free surface.

Recently, Gray \& Ancey (2009) found a regime in which grains came to rest by both basal deposition and shock wave propagation. Their experiments were performed in a Hele-Shaw cell with a $3 \mathrm{~mm}$ gap and an approximately 50:50 mix by volume of 500$600 \mu \mathrm{m}$ white sugar crystals and $210-420 \mu \mathrm{m}$ spherical dark iron powder. A digital photo of an avalanche front propagating down the surface of the pile is shown in figure $9(a)$. The large white particles that form part of the current flow have been highlighted by overlaying a darkened image of the initial deposit. The flow front is entirely composed of large particles, but within the interior a layer of small particles is sandwiched between large grains at the free surface and a carpet of deposited coarse particles at the base. Gray \& Ancey (2009) suggested that there was a 'segregation mobility feedback effect' in which large particles that were sheared to the flow front, experienced greater resistance to motion and were deposited to enable the avalanche to continue to propagate downslope.

Within the interior the deposition stops and a steady uniform flow develops. Although there are some exponentially small motions deeper down in the pile (Jesuthasan, Baliga \& Savage 2006), the main transition from the flowing avalanche to an essentially stationary body occurs just above the penultimate layer of large particles. The avalanche is therefore still inversely graded even though the particle size distribution looks like a small particle sandwich. When the flow is brought to rest by 
(a)

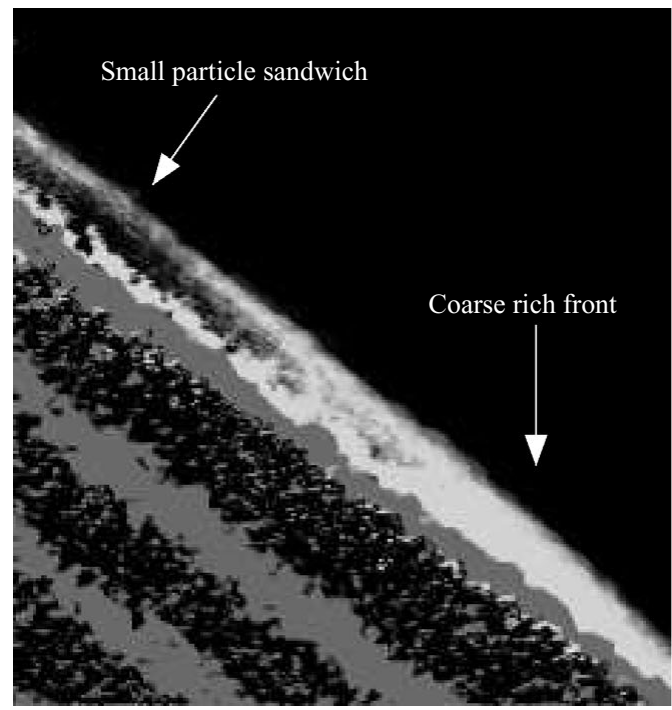

(b)

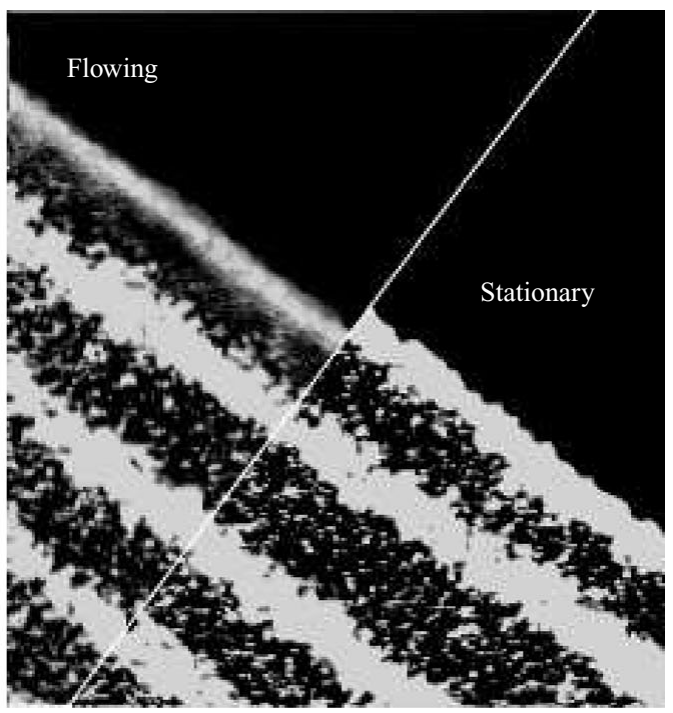

FIGURE 9. Two composite images showing $(a)$ the propagation of an avalanche front down the surface of a stratified pile and $(b)$ the jump in the avalanche thickness and inversely graded interface height (across the white line) as a flowing avalanche is brought to rest by an upslope propagating granular bore. The images all come from the same experiment and are constructed from panels $(a),(g),(o)$ and $(t)$ shown in figure 1 of Gray \& Ancey (2009). The white grains are $500-600 \mu \mathrm{m}$ sugar crystals and the region with a slightly mottled appearance consists of $210-420 \mu \mathrm{m}$ dark iron spheres. A shaded overlay of the initial deposit has been used in $(a)$ to highlight large grains that form part of the current flow. Each image is $36.1 \mathrm{~mm}$ wide by $38.2 \mathrm{~mm}$ high and an animation showing the deposition of two avalanches is available with the online version of this paper (supplementary movie 3 ).

the upslope propagation of a granular bore, as shown in figure $9(b)$, the avalanche increases in thickness and the inverse grading is preserved in the final deposit.

The stratification pattern experiments shown in figure $9(a, b)$ are interesting, because even though the parent flow is inversely graded in both cases they show that two qualitatively different mechanisms for deposition give rise to completely opposite gradings in the final deposit. In figure $9(a)$ basal deposition close to the flow front leads to a normally graded deposit that is overrun by the avalanche, while in figure $9(b)$ a diffuse shock wave brings the grains to rest and preserves the inverse grading in the final deposit. It is of considerable interest to see whether the transport theory can capture these phenomena.

\subsection{A travelling wave solution near a depositing front}

In order to apply the large particle transport equation to a depositing avalanche front, the theory must first be generalized to include the effects of basal deposition. Assuming that the flow is shallow, the basal kinematic condition (e.g. Gray 2001) becomes

$$
\frac{\partial b}{\partial t}+u_{b} \frac{\partial b}{\partial x}-w_{b}=d \quad \text { at } \quad z=b(x, t),
$$

where $\left(u_{b}, w_{b}\right)$ are the velocity components at the base of the avalanche and $d$ is the normal deposition rate per unit area per unit time. Depth integrating the incompressibility condition (2.2) and the segregation equation (2.10) through the 
avalanche depth and substituting the kinematic condition (4.1) yields

$$
\begin{aligned}
\frac{\partial h}{\partial t}+\frac{\partial}{\partial x}(h \bar{u}) & =-d, \\
\frac{\partial \eta}{\partial t}+\frac{\partial}{\partial x}(\eta \bar{u})-\frac{\partial}{\partial x}\left((1-\alpha) \bar{u} \eta\left(1-\frac{\eta}{h}\right)\right) & =-\phi_{b} d,
\end{aligned}
$$

where $\phi_{b}$ is the small particle concentration at the base of the avalanche. The inversely graded concentration distribution (2.15) implies that there are either (i) large particles at the bottom of the flow $\left(\phi_{b}=0\right)$ and (4.3) is trivially satisfied by

$$
\eta=0
$$

or (ii) there are small particles $\left(\phi_{b}=1\right)$ and the transport equation reduces to

$$
\frac{\partial \eta}{\partial t}+\frac{\partial}{\partial x}(\eta \bar{u})-\frac{\partial}{\partial x}\left((1-\alpha) \bar{u} \eta\left(1-\frac{\eta}{h}\right)\right)=-d .
$$

The deposition term on the right-hand side of (4.5) is the same as that in (4.2), which is consistent with the notion that all the particles that are deposited are small when there are small particles at the base of the flow.

Motivated by the observations in figure 9(a) and following Gray \& Ancey (2009), a travelling wave solution is sought in a frame moving downslope with speed $u_{F}$. Using the transformation

$$
\xi=x-u_{F} t, \quad \tau=t,
$$

and assuming that $u_{b}=w_{b}=0$, the kinematic condition (4.1) implies that the deposition rate $d=-u_{F} \partial b / \partial \xi$. Substituting this into the depth-averaged mass balance equation (4.2) and the transport equation (4.5) implies that

$$
\begin{aligned}
\frac{\partial}{\partial \xi}\left(h\left(\bar{u}-u_{F}\right)-u_{F} b\right) & =0, \\
\frac{\partial}{\partial \xi}\left(-\eta u_{F}+\bar{u} \frac{\eta^{2}}{h}-u_{F} b\right) & =0 .
\end{aligned}
$$

The first of these equations can be integrated subject to the condition that $h=b=0$ at the avalanche front to give

$$
b=\lambda h, \quad \text { where } \quad \lambda=\frac{\bar{u}-u_{F}}{u_{F}} .
$$

When there is no deposition the depth-averaged mass balance equation (4.7) implies that $\bar{u}$ is equal to the front velocity $u_{F}$ (Pouliquen 1999b). Problems with deposition are notoriously difficult and there is presently no complete theory to determine the deposition rate $d$. One approach is to prescribe $d$ (e.g. Doyle et al. 2007) based on experimental observations (Lajeunesse, Mangeney-Castelnau \& Vilotte 2004; Lube et al. 2004). This paper follows Gray \& Ancey (2009) who instead prescribed the depth-averaged velocity

$$
\bar{u}=(1+\lambda) u_{F},
$$

to be constant throughout the avalanche.

Steady uniform flows develop on rough beds over a range of angles $\zeta_{1} \leqslant \zeta \leqslant \zeta_{2}$, which Pouliquen (1999a) and Pouliquen \& Forterre (2002) used to derive a new friction law appropriate for these surfaces. In addition, rough beds also support travelling flow fronts, which propagate downslope at constant speed and have a constant depth-averaged velocity throughout the flow. Their free surface shape $s(\xi)$ 


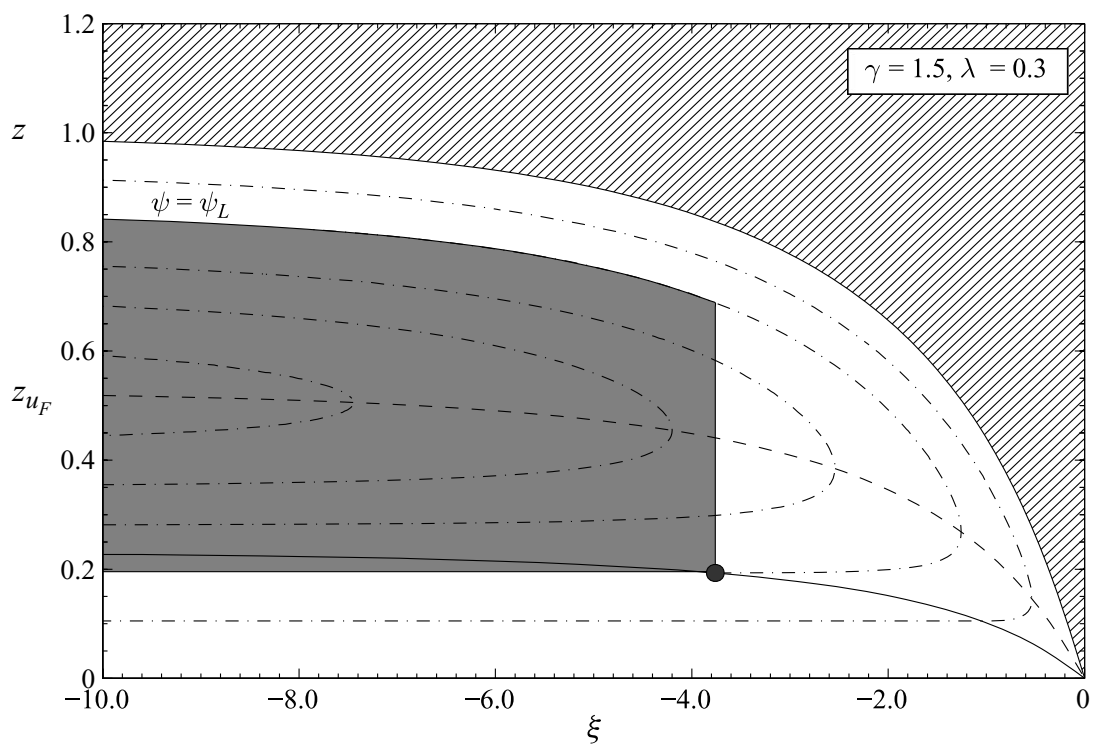

FiguRE 10. The solution of the transport equation at a depositional flow front. The small particle region is shown in grey and the large particle region is shown in white. The free surface $s$ and basal deposition surface $b$ are shown with solid black lines, the hatched region is empty and the dot-dashed lines show the particles paths of the bulk flow. The material above the no-mean flow line $z_{u_{F}}$ (dashed line) moves towards the front, and that below it moves backwards.

can then be calculated from the depth-averaged downslope momentum balance (2.9) using the rough bed friction law. Pouliquen (1999b) did this numerically using the exponential form of his friction law for rough beds (Pouliquen 1999a). More recently, Gray \& Ancey (2009) used Pouliquen \& Forterre's (2002) modified reciprocal form of the friction law to derive the exact solution

$$
\xi=(1+\gamma) s+\frac{(1+\gamma)^{2}}{3 \gamma}\left[\ln \left(\frac{(1-\sqrt{s})^{2}}{s+\sqrt{s}+1}\right)+2 \sqrt{3} \operatorname{atan}\left(\frac{1+2 \sqrt{s}}{\sqrt{3}}\right)-\frac{\pi}{\sqrt{3}}\right],
$$

where

$$
\gamma=\frac{\tan \zeta_{2}-\tan \zeta_{1}}{\tan \zeta-\tan \zeta_{1}}-1
$$

and $\zeta_{2}$ and $\zeta_{1}$ are the maximum and minimum angles for which steady uniform flows are observed. The solution is illustrated in figure 10, which shows that the avalanche front is located at $\xi=0$ and that as $\xi \longrightarrow-\infty$ the free-surface height $s \longrightarrow 1$. This solution implicitly assumes that the maximum free-surface height $H$ is used to scale the vertical coordinate in (2.23) and that the horizontal length scale $L=H /\left(\tan \zeta_{2}-\tan \zeta_{1}\right)$. The results that follow are not dependent on the precise form of the avalanche front and Gray \& Ancey's (2009) profile is used primarily to enable direct comparison with their two-dimensional solutions for the small particle concentration.

In order to solve the two-dimensional segregation equation (2.27) near a depositing avalanche front, Gray \& Ancey (2009) mapped the $(\xi, z)$ coordinates into a set of streamfunction coordinates $(\xi, \psi)$ that linearized the problem. The streamfunction coordinates are also useful for solving the transport equation (4.3) and are constructed 
by calculating the velocity integral

$$
\psi=\int_{0}^{z} u\left(\xi, z^{\prime}\right)-u_{F} \mathrm{~d} z^{\prime} .
$$

Assuming that the downslope velocity is linear with depth and zero at the base

$$
u=\left\{\begin{array}{cc}
2 \bar{u}\left(\frac{z-b}{h}\right), & b \leqslant z \leqslant s, \\
0, & 0 \leqslant z \leqslant b,
\end{array}\right.
$$

the streamfunction

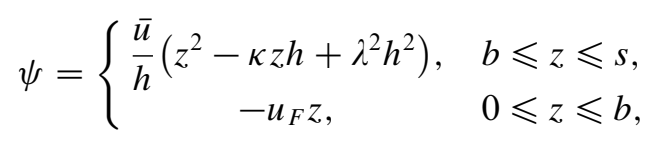

where $\kappa$ is a function of the deposition parameter $\lambda$

$$
\kappa=2 \lambda+\frac{1}{1+\lambda} .
$$

The streamfunction $\psi$ is zero at the surface and base of the flow and attains a local minimum on the no-net flow line at $z=z_{u_{F}}$. This marks the point where the bulk velocity $u$ is equal to the front velocity $u_{F}$ and is shown with a dashed line in figure 10 . The dot-dashed lines are used to illustrate lines of constant $\psi$, which are also particle paths of the bulk flow field. Substituting the definition $z=\eta+b$ into the first equation in (4.15) implies that the streamfunction $\psi$ can also be expressed as

$$
\psi=-\eta u_{F}+\bar{u} \frac{\eta^{2}}{h}-u_{F} b,
$$

which is of precisely the same form as the term in round brackets in (4.8). The streamfunction coordinates $(\xi, \psi)$ therefore also linearize the one-dimensional transport equation, which becomes

$$
\frac{\partial \psi}{\partial \xi}=0 \quad \Rightarrow \quad \psi=\psi_{L} .
$$

It follows from (4.4) and (4.18) that either the shock interface height $\eta$ equals zero or it lies along a constant streamline $\psi_{L}$ of the bulk flow field. Physically these two solutions correspond to the coarse-grained flow front and the incoming inversely graded avalanche. The solutions are exactly the same as in the two-dimensional theory, where they are joined together by a breaking size segregation wave that lies at a unique position behind the flow front (Gray \& Ancey 2009). In the one-dimensional transport theory the solutions are instead joined by a shock in $\eta$ that satisfies the jump condition (3.8). Assuming that interface height on the forward side of the shock, $\eta_{2}$, is zero and that the shock travels at the same speed as the front, the rearward interface height

$$
\eta_{1}=\frac{h u_{F}}{\bar{u}} .
$$

Substituting (4.18) and (4.19) into the streamfunction (4.17) implies that the shock is located at the point where the basal topography is of height

$$
b_{\text {shock }}=-\frac{\psi_{L}}{u_{F}},
$$


(a)

(b)
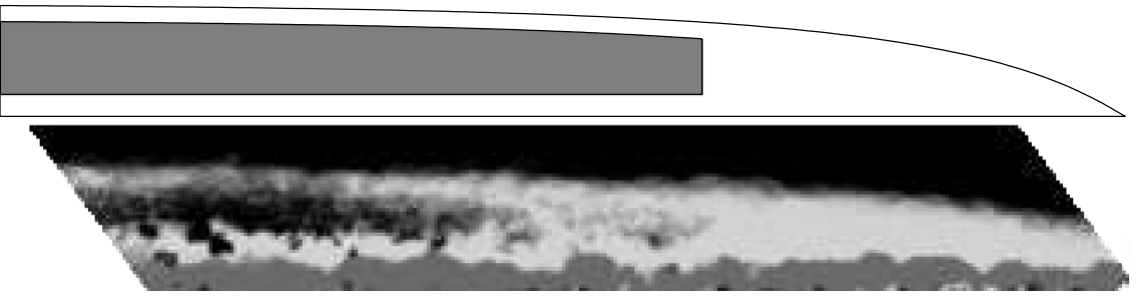

FiguRE 11. The solution of the transport equation at a depositional flow front is plotted in a more realistic aspect ratio $(a)$ and a rotated and cropped image of the experimental avalanche front of figure $9(a)$ is shown for comparison $(b)$. Note that both the exact solution and experiment have a large rich flow front that is connected to a small particle sandwich in the interior.

which is indicated by the black dot in figure 10. This also corresponds to the position where the large particle path $\psi_{L}$ intersects with the basal topography, which can easily be shown by substituting $\eta=0$ into the streamfunction (4.17). The shock therefore connects the two branches of the $\psi_{L}$ streamline at the point where it intersects the basal topography as shown in figure 10 .

Ahead of the shock lies the coarse-grained front and behind it lies the inversely graded avalanche. It follows that large particles are deposited ahead of the shock and small particles are laid down behind the shock. Even though the avalanche is inversely graded, the combination of deposition at the base and continued flow over the top implies the grains are deposited in a normally graded configuration separated by a contact discontinuity at height

$$
z=b_{\text {shock }}
$$

as shown in figure 10. The complete solution is also shown in a more realistic aspect ratio in figure $11(a)$ together with a rotated close-up view of the experimental flow front in figure 11(b) for comparison. Although the representation of the particle size distribution is relatively crude, this simple theory is able to reproduce the broad features of the flow, which include a coarse rich flow front and a layer of small particles that is sandwiched between two layers of large particles as observed in the experiment.

The solution relies on all the large particles that reach the front being deposited. There are therefore no solutions if the incoming shock lies on a particle path that is recirculated within the avalanche. The critical curve, between solutions and no solutions, corresponds to the particle path that starts at minus infinity at the height where the velocity is twice the front speed $u_{F}$. This intersects the basal topography at a height $b=\lambda /(1+\lambda)$ as $\xi \longrightarrow-\infty$. The value of the streamfunction on this path is

$$
\psi_{2 u_{F}}^{\infty}=-u_{F} \frac{\lambda}{1+\lambda},
$$

and it follows that for

$$
\begin{aligned}
\psi_{2 u_{F}}^{\infty} \leqslant \psi_{L} \leqslant 0: & b_{\text {shock }}=\frac{-\psi_{L}}{u_{F}}, \\
\psi_{L}<\psi_{2 u_{F}}^{\infty}: & \text { no solution. }
\end{aligned}
$$

In the limit as $S_{r} \longrightarrow \infty$ the breaking size segregation wave in the full two-dimensional segregation theory (Gray \& Ancey 2009) collapses onto a shock located at $s=$ $-\psi_{L}(1+\lambda) /\left(u_{F} \lambda\right)$ (see their equation (3.39)). Using the identity $b=\lambda s /(1+\lambda)$ it 
is easy to recover (4.23). The one-dimensional transport equation (4.3) and the full two-dimensional segregation theory are therefore exactly equivalent in the limit as $S_{r} \longrightarrow \infty$. One of the major advances that Gray \& Ancey (2009) made was the construction of an exact solution for the large particle paths near the avalanche front. They showed that the particles that entered into the breaking size segregation wave were recirculated, forming closed loops (see their figure 13), while all the large particles that were sheared to the flow front were overrun and deposited. In the limit as $S_{r} \longrightarrow \infty$ the width of the breaking wave tends to zero, and the closed loops have to jump at the shock. Transport theory therefore implies that those large particles that are not deposited are still recirculated at the flow front.

\subsection{Deposition of a stripe by a granular bore}

Consider now the problem of how the flowing particles at the surface of the heap are brought to rest by an upslope propagating granular bore (Gray \& Hutter 1997), which is a critical process in the formation of stratified deposits. The increase in the avalanche thickness and the speed of the propagation of the bore are controlled by the depth-averaged mass and momentum jump conditions (e.g. Gray et al. 2003), while the jump in the inversely graded interface height $\eta$ is controlled by the transport jump condition (3.8). These can be summarized by the relations

$$
\begin{aligned}
h\left(\bar{u}-v_{n}\right) & =0, \\
h u\left(u-v_{n}\right)+\frac{1}{2} h^{2} \varepsilon \cos \zeta & =0, \\
\eta\left(\bar{u}-v_{n}\right)-(1-\alpha) \bar{u} \eta(1-\eta / h) & =0,
\end{aligned}
$$

where the velocity magnitude is assumed to scale as $U=\sqrt{g L}$. An interesting observation from figure $9(b)$ is that the velocity shear within the avalanche implies that there is a much higher mass flux of particles near the free surface than at the base of the flow. As a result, when the grains are brought to rest the upper layer of large particles expands proportionately more than the layer of fines. This can be seen most clearly in the online video that accompanies this paper and is shown schematically in figure 12 . The stationary layers below the avalanche are unaffected by the bore.

This subtle effect is captured by the transport jump condition (4.27). Assuming that the grains are stationary on the downstream side of the shock $\left(\bar{u}_{2}=0\right)$ and using the mass jump condition (4.25), the height of the interface on the downstream side of the shock can be expressed as

$$
\eta_{2}=\eta_{1}+\left(\frac{h_{2}}{h_{1}}-1\right)\left(\alpha \eta_{1}+(1-\alpha) \frac{\eta_{1}^{2}}{h_{1}}\right) .
$$

This implies that the interface height $\eta_{2}$ will be greater than the incoming height $\eta_{1}$ provided $h_{2}>h_{1}$. Dividing (4.28) by $h_{2}$ and using the definition $\eta=h \bar{\phi}$ the depthaveraged small particle concentration on the downslope side is

$$
\bar{\phi}_{2}=\bar{\phi}_{1}-\left(1-\frac{h_{1}}{h_{2}}\right)(1-\alpha)\left(\bar{\phi}_{1}-\bar{\phi}_{1}^{2}\right) .
$$

The concentration $\bar{\phi}_{2}$ is therefore less than the upslope concentration $\bar{\phi}_{1}$, provided $h_{2}>h_{1}$ and the velocity profile is not plug-like $(\alpha \neq 1)$. The transport theory predicts that the upper layer of large particles will thicken more than the small particle layer at the base, provided there is velocity shear.

As all the necessary variables can be measured from figure $9(b)$ it is interesting to test the accuracy of the predictions. Recalling that the base of the avalanche lies just 


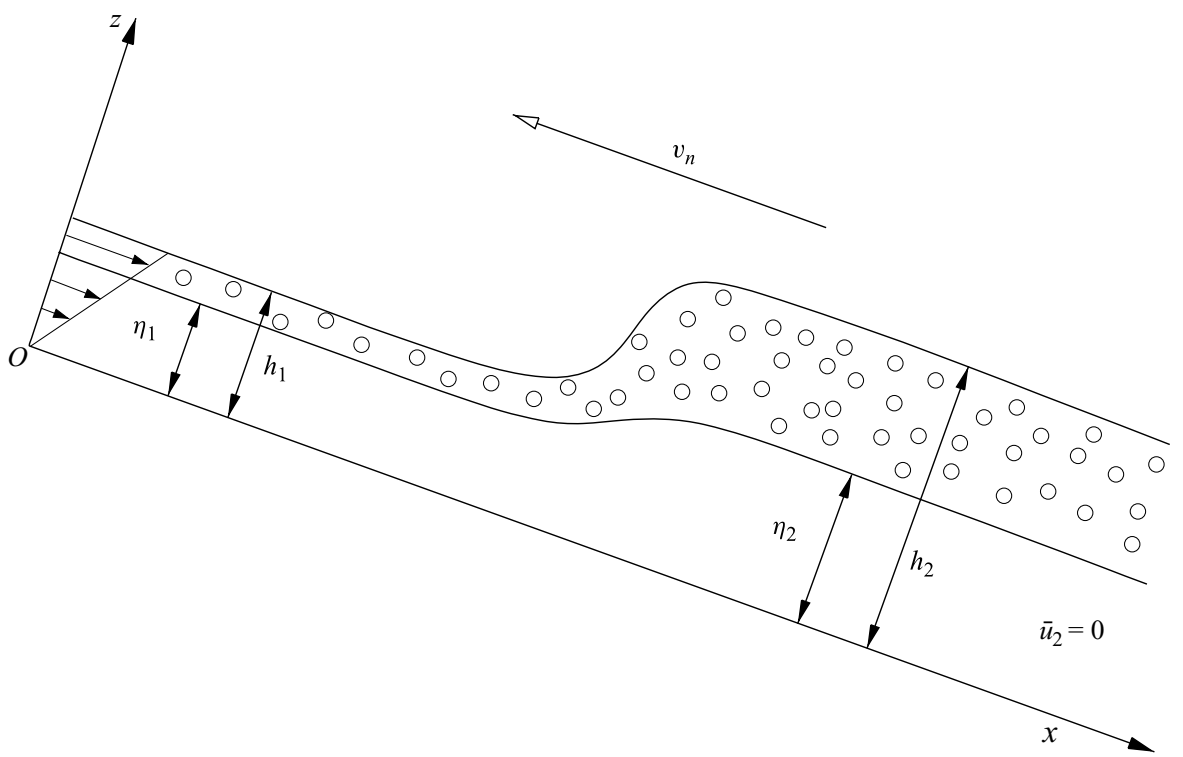

FiguRE 12. A schematic diagram showing how the layer of large particles expands more than the layer of fines as it is brought to rest by a normal shock wave propagating upstream with speed $v_{n}$. Prior to the shock the avalanche is sheared through its depth $h_{1}$, and the small particle layer is of thickness $\eta_{1}$. After the shock has passed, the grains are stationary with a total thickness $h_{2}$ and a small particle depth $\eta_{2}$.

above the penultimate layer of large grains in the deposit, it follows that upstream of the bore

$$
h_{1}=3.8 \mathrm{~mm}, \quad \eta_{1}=2.53 \mathrm{~mm} \quad \Rightarrow \quad \bar{\phi}_{1}=0.66,
$$

and downstream of it

$$
h_{2}=5.38 \mathrm{~mm}, \quad \eta_{2}=3.16 \mathrm{~mm} \quad \Rightarrow \quad \bar{\phi}_{2}=0.58 .
$$

Substituting the measured values of $h_{1}, \eta_{1}$ and $h_{2}$ into (4.29) and assuming that there is simple shear $(\alpha=0)$, the depth-averaged small particle concentration in the stationary deposit is

$$
\bar{\phi}_{2}=0.59 \text {, }
$$

which is within $3 \%$ of the measured value of 0.58 . Despite its simplicity, the onedimensional depth-averaged transport theory is able to capture these subtle features of the flow.

This effect can also be picked up by the two-dimensional segregation theory. The solution is assumed to be inversely graded on either side of the granular bore and to have stable inversely graded shocks at heights $\eta_{1}$ and $\eta_{2}$, respectively. The mass balance of small particles implies that

$$
\int_{0}^{\eta_{1}} \rho_{1}\left(u_{1}-v_{n}\right) \mathrm{d} z=\int_{0}^{\eta_{2}} \rho_{2}\left(u_{2}-v_{n}\right) \mathrm{d} z .
$$

Assuming that the changes in density are negligible and that the bulk velocity $u_{1}=\alpha \bar{u}_{1}+2(1-\alpha) \bar{u}_{1} z / h_{1}$ upstream of the bore and $u_{2}=0$ downstream of it, the 
integral (4.33) implies that the downstream interface height

$$
\eta_{2}=\eta_{1}+\alpha\left(\frac{\bar{u}_{1}}{-v_{n}}\right) \eta_{1}+(1-\alpha)\left(\frac{\bar{u}_{1}}{-v_{n}}\right) \frac{\eta_{1}^{2}}{h_{1}} .
$$

This is equivalent to the jump condition (4.27) and can be put into the same form as (4.28) by using the mass jump relation (4.25). The two-dimensional segregation theory therefore makes exactly the same prediction as the depth-integrated large particle transport equation.

\section{Large particle accumulation at a non-depositing flow front}

An exact steady-state solution for the particle size distribution near a steadily depositing flow front was constructed in $\S 4.1$, by ensuring that all the large particles that reached the front were deposited. In this section a related problem is investigated in which there is no deposition and instead the large particles accumulate at a steadily propagating avalanche front. Transforming to a frame moving with the front speed $u_{F}$,

$$
\xi=x-u_{F} t, \quad \tau=t,
$$

implies that the non-dimensional depth-averaged mass balance (2.7) becomes

$$
\frac{\partial h}{\partial \tau}+\frac{\partial}{\partial \xi}\left(h\left(\bar{u}-u_{F}\right)\right)=0 .
$$

Assuming that the shape of the flow front is independent of time implies that either

$$
h=0, \quad \text { or } \quad \bar{u}=u_{F} .
$$

For non-trivial solutions the depth-integrated velocity is therefore constant throughout the avalanche. This is a direct consequence of mass balance rather than a model assumption, as in (4.10) of the depositing flow problem. The coordinate transformation (5.1) and the time independence of the flow front (5.3) imply that the large particle transport equation (2.24) reduces to

$$
\frac{\partial \eta}{\partial \tau}-\frac{\partial}{\partial \xi}\left((1-\alpha) u_{F} \eta\left(1-\frac{\eta}{h}\right)\right)=0 .
$$

Since $u_{F}$ is constant this equation can be expanded to give

$$
\frac{\partial \eta}{\partial \tau}+(1-\alpha) u_{F}\left(\frac{2 \eta}{h}-1\right) \frac{\partial \eta}{\partial \xi}=(1-\alpha) u_{F} \frac{\eta^{2}}{h^{2}} \frac{\mathrm{d} h}{\mathrm{~d} \xi},
$$

which can be solved by the method of characteristics. Introducing a parameter $\lambda$ running along the characteristic curves the corresponding characteristic differential equations are

$$
\frac{\mathrm{d} \tau}{\mathrm{d} \lambda}=1, \quad \frac{\mathrm{d} \xi}{\mathrm{d} \lambda}=(1-\alpha) u_{F}\left(\frac{2 \eta}{h}-1\right), \quad \frac{\mathrm{d} \eta}{\mathrm{d} \lambda}=(1-\alpha) u_{F} \frac{\eta^{2}}{h^{2}} \frac{\mathrm{d} h}{\mathrm{~d} \xi} .
$$

The second and third of these equations uncouple from the first and imply that

$$
\frac{\mathrm{d}}{\mathrm{d} \xi}\left(-(1-\alpha) u_{F} \eta\left(1-\frac{\eta}{h}\right)\right)=0 .
$$

Assuming that the topography is flat $(b=0)$ and that the downslope velocity profile through the depth of the avalanche is given by (2.17), the streamfunction coordinate 


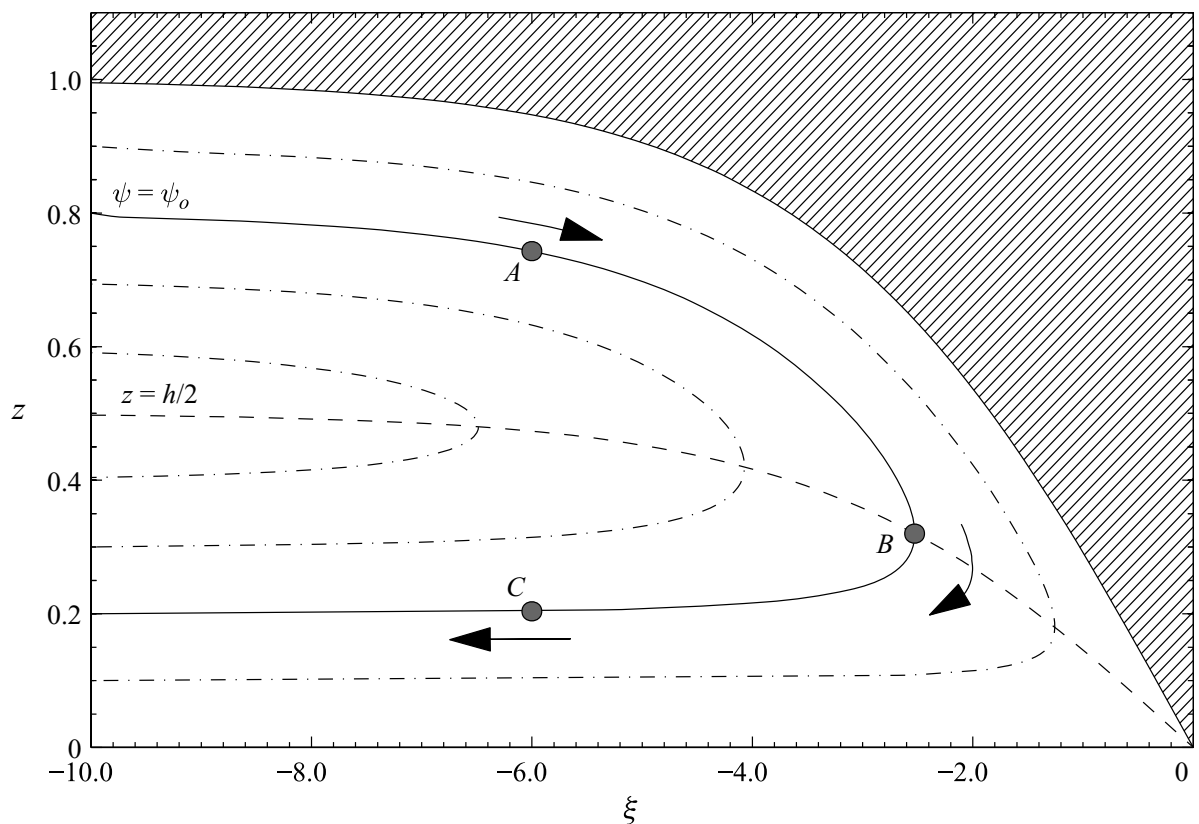

FiguRE 13. A vertical section through the front of an avalanche showing the bulk streamfunction $\psi$ using dot-dashed lines. The streamline corresponding to $\psi=\psi_{o}$ is highlighted as a solid line. The hatched region above $z=h$ is grain-free and the dashed line at $z=h / 2$ marks the line of no-net flow relative to the moving front. The characteristics propagate information about the interface height $\eta$ along a fixed streamline $\psi_{o}$. Point $A$ moves towards the front before reaching the no-mean flow line at point $B$ and moving backwards towards point $C$. An interface that initially lies at point $\mathrm{C}$ will move backwards immediately.

(4.13) becomes

$$
\psi=-(1-\alpha) u_{F} \eta\left(1-\frac{\eta}{h}\right),
$$

and linearizes (5.7). Substituting (5.8) into (5.7) and integrating implies that

$$
\psi=\psi_{o}=(1-\alpha) u_{F} \Psi_{o},
$$

is a constant. The characteristic curves therefore propagate along the streamlines of the bulk flow. The streamfunction $\psi$ is zero at the surface and base of the avalanche, and it attains a local minimum $\psi_{\min }=-(1-\alpha) u_{F} h / 4$ at $z=h / 2$. Provided $\psi_{\min } \leqslant \psi_{o} \leqslant 0$, there are two solutions for the shock height $\eta$ for any given value of $h$

$$
\eta= \begin{cases}\frac{h+\sqrt{h^{2}+4 \Psi_{o} h}}{2}, & h / 2 \leqslant \eta \leqslant h, \\ \frac{h-\sqrt{h^{2}+4 \Psi_{o} h}}{2}, & 0 \leqslant \eta \leqslant h / 2,\end{cases}
$$

where the constant $\Psi_{o}$ is defined in (5.9). The streamlines close to an avalanche front are illustrated schematically in figure 13. Each line of constant $\psi=\psi_{o}$ carries information about the position of the interface $\eta$ along it. If $\eta>h / 2$ then information propagates towards the flow front, while if $\eta<h / 2$ information is carried back away from it. To calculate how quickly this information propagates along each streamline it is necessary to solve the first and second equations in (5.6) using (5.10), which yields 
an ordinary differential equation for the position $\xi$ as a function of time

$$
\frac{\mathrm{d} \xi}{\mathrm{d} \tau}= \pm(1-\alpha) u_{F} \sqrt{1+\frac{4 \Psi_{o}}{h(\xi)}}
$$

The plus/minus sign on the right-hand side is dependent on whether the interface is on the upper or lower branch of the streamline in (5.10). At the surface and base of the flow (5.11) can be integrated directly, since $\Psi_{o}$ is zero. Assuming that the interface initially lies at point $\xi_{o}$ on the free surface $h$, its position at later times is given by

$$
\xi= \begin{cases}\xi_{o}+(1-\alpha) u_{F} \tau, & \tau \leqslant \tau_{\text {turn }}, \\ -\xi_{o}-(1-\alpha) u_{F} \tau, & \tau>\tau_{\text {turn }},\end{cases}
$$

where

$$
\tau_{\text {turn }}=-\frac{\xi_{o}}{(1-\alpha) u_{F}}
$$

is the time that the information reaches $\xi=0$ and starts travelling back along the line $z=0$. Conversely, a point that starts at the base at point $\xi_{o}$ stays on the base for all time and its horizontal position

$$
\xi=\xi_{o}-(1-\alpha) u_{F} \tau .
$$

To determine the position of the interface on internal streamlines, (5.11) must be solved for a given thickness distribution $h(\xi)$. It is possible to solve the problem numerically using the exact solution (4.11) for Pouliquen \& Forterre's (2002) reciprocal form of the friction law. However, it is instructive to use a simple profile that allows for exact integration, e.g.

$$
h=-\tanh (\beta \xi), \quad x \in(-\infty, 0],
$$

where $\beta$ is the inverse length scale of the front. By using the chain rule and (5.15) to substitute for $\mathrm{d} \xi / \mathrm{d} h,(5.11)$ can be written as the separable ordinary differential equation

$$
\pm(1-\alpha) \beta u_{F} \frac{\mathrm{d} \tau}{\mathrm{d} h}=\left(\frac{-1}{1-h^{2}}\right) \sqrt{\frac{h}{h+4 \Psi_{o}}} .
$$

If the initial position of the interface $\left(\xi_{o}, \eta_{o}\right)$ lies above the no-mean flow line, $\eta_{o}>h_{o} / 2$, where $h_{o}=h\left(\xi_{o}\right)$, then it is transported towards the flow front along the streamline $\Psi_{o}=-\eta_{o}\left(1-\eta_{o} / h_{o}\right)$, as for point $\mathrm{A}$ in figure 13 . The current position is computed by integrating (5.16) with the positive root to give

$$
(1-\alpha) \beta u_{F} \tau=I(h)-I\left(h_{o}\right),
$$

where the function

$$
I(h)=\frac{1}{2 \sqrt{1-4 \Psi_{o}}} \ln \left(\frac{1}{2 A}\right)+\frac{1}{2 \sqrt{1+4 \Psi_{o}}} \ln \left(\frac{1}{2 B}\right),
$$

and

$$
\begin{aligned}
& A=\frac{2 \Psi_{o}-2 \Psi_{o} h+h-\sqrt{1-4 \Psi_{o}} \sqrt{h^{2}+4 \Psi_{o} h}}{1+h}, \\
& B=\frac{2 \Psi_{o}+2 \Psi_{o} h+h+\sqrt{1+4 \Psi_{o}} \sqrt{h^{2}+4 \Psi_{o} h}}{1-h} .
\end{aligned}
$$


For a given time $\tau$ the height of the avalanche at the current interface position $h$ can be determined by iterating for $h \in\left[h_{\text {turn }}, 1\right]$ using (5.17); the interface height $\eta$ lies on the upper branch of the streamline, which is determined by the positive root in (5.10). The interface moves along the streamline until it reaches the no-mean flow line at point $\mathrm{B}$ in figure 13 at position $\xi_{\text {turn }}$ and time $\tau_{\text {turn }}$, where

$$
\begin{aligned}
\xi_{\text {turn }} & =\tanh ^{-1}\left(4 \Psi_{o}\right) / \beta, \\
(1-\alpha) \beta u_{F} \tau_{\text {turn }} & =I\left(h_{\text {turn }}\right)-I\left(h_{o}\right),
\end{aligned}
$$

and $h_{\text {turn }}=h\left(\xi_{\text {turn }}\right)$. For $\tau>\tau_{\text {turn }}$ the interface moves back along the lower branch of the streamline $\Psi_{o}$ towards point C. Integrating along the negative branch of (5.16) and applying the condition that $h=h_{\text {turn }}$ at $\tau=\tau_{\text {turn }}$ implies that for $\tau>\tau_{\text {turn }}$

$$
(1-\alpha) \beta u_{F}\left(\tau_{\text {turn }}-\tau\right)=I(h)-I\left(h_{\text {turn }}\right),
$$

which can alternatively be expressed as

$$
(1-\alpha) \beta u_{F}\left(2 \tau_{\text {turn }}-\tau\right)=I(h)-I\left(h_{o}\right),
$$

using (5.22). For $\eta_{o}>h_{o} / 2$ and $\tau>\tau_{\text {turn }}$ the position of the interface on the streamline $\Psi_{o}$ can therefore be found by iterating for $h \in\left(0, h_{\text {turn }}\right)$ using (5.24) and then substituting the result into the lower branch of (5.10). The final case, in which $\eta_{o} \leqslant h_{0} / 2$, occurs when the interface starts on the lower branch of the streamline, as for point $\mathrm{C}$ in figure 13, and moves away from the front. Integrating (5.16) with the negative root subject to the initial condition that $h(0)=h_{o}$ implies that the avalanche thickness $h$ can be found by iterating for $h \in\left(0, h_{\text {turn }}\right)$ using

$$
-(1-\alpha) \beta u_{F} \tau=I(h)-I\left(h_{o}\right),
$$

and $\eta$ is given by substituting the result into the negative branch of (5.10). In each of the three cases (5.17), (5.24) and (5.25) the horizontal position $\xi$ of the interface is given by inverting (5.15).

Figure 14 shows a specific example of the solution that illustrates how large particles are transported to the flow front and then accumulate there. At $\tau=0$ the front is initially composed of all small particles in the region $\xi \in(-10,0)$. For $\tau>0$ large particles are fed in at the boundary, at $\xi_{0}=-10$, above an interface which lies at $\eta=0.8 h_{0}$. The initial discontinuity forms an expansion fan that is advected downstream towards the front, leaving behind a steady interface that lies along a streamline. For parameters $\alpha=0$ and $\bar{u}=1$, the fastest moving part of the interface, which lies at the free surface, reaches the flow front $\xi=0$ at $\tau=10$. After this time it moves back along the base. The interior interface is also swept along the streamlines and becomes triple valued after $\tau=10$, with the shape of the interface indicated by a dotted line in figure 14. As the interface breaks, a shock is formed and its position is determined by a shock fitting procedure (e.g. Whitham 1974). The shock forms at $\xi=0$ at $\tau=10$ and grows in amplitude with increasing time as it propagates backwards relative to the moving front. It separates a coarse grained flow front from the inversely graded avalanche behind, which continuously supplies more large grains that are then recirculated at the front. It is this type of two-dimensional configuration that, when appropriately coupled to the bulk flow, is likely to be unstable in threedimensions, spontaneously producing lateral levees as in figure 2 , in which the resistive large grains are removed by lateral transport rather than by basal deposition. 

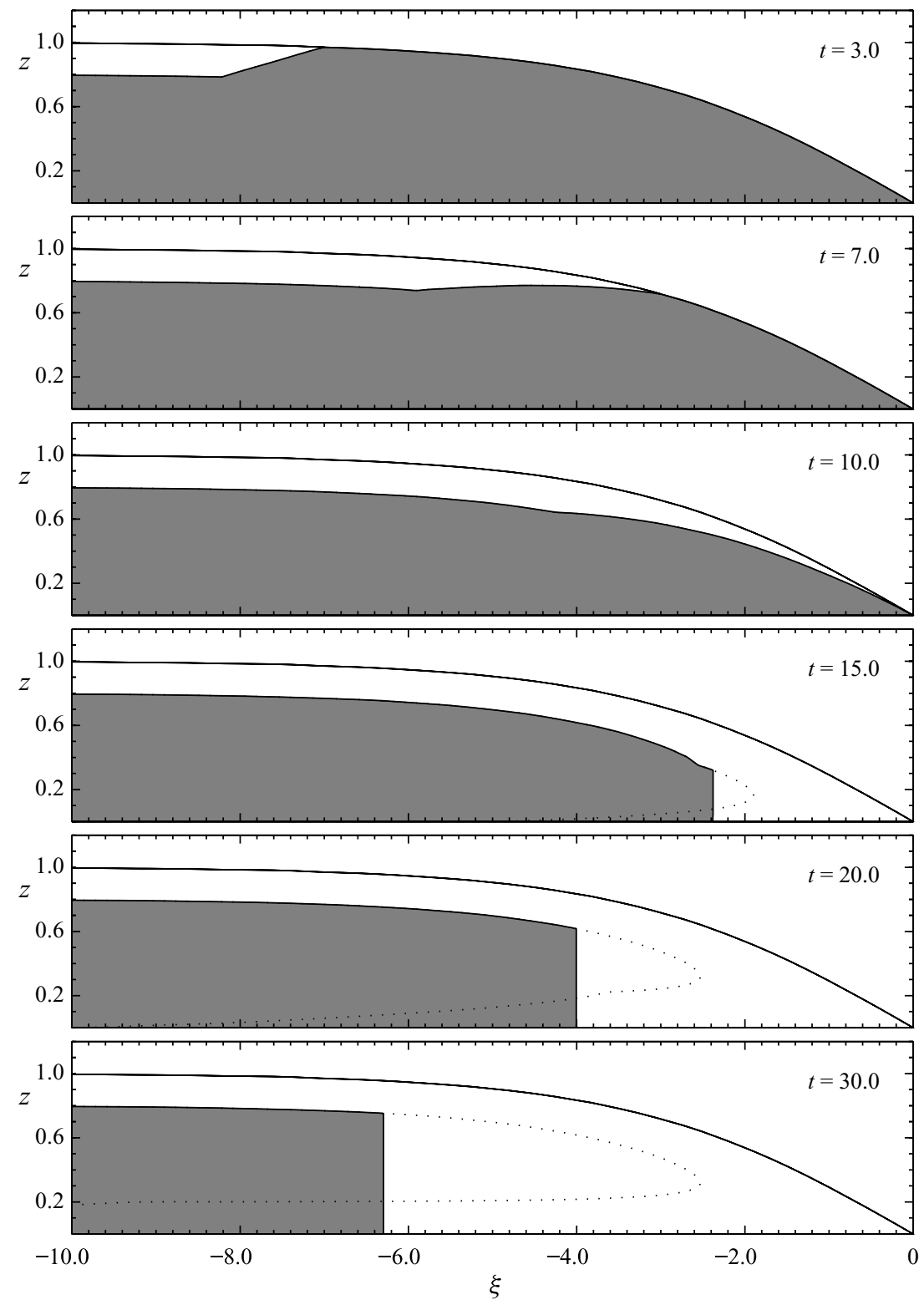

FIGURE 14. A series of vertical sections through the avalanche showing how large particles are transported towards the flow front and then accumulate there for parameters $\alpha=0$ and $\bar{u}=1$. At $\tau=0$, small (grey) particles occupy the region $(-10,0)$ and subsequently large particles (white) are input at $\xi_{0}=-10$ above $0.8 h_{0}$. The avalanche free surface $h=-\tanh (\beta \xi)$ with $\beta=0.3$ is shown by a solid line. The large particles reach the front at $\tau=10$ and the interface (dotted line) becomes triple valued. A shock fitting procedure is used to determine the position of the shock, which propagates backwards relative to the front with increasing time. An animated version is available with the online version of this paper (supplementary movie 4).

\section{Conclusions}

Particle size segregation and velocity shear are the two key mechanisms that transport large particles towards an avalanche front and then allow those large 
particles that reach it to recirculate and accumulate there. In this paper the segregation-remixing equation has been integrated through the avalanche depth, assuming that (i) the avalanche is always inversely graded and (ii) the velocity shear is linear with depth, to derive a depth-averaged large particle transport equation (2.19) that can model these effects. This may be viewed as the simplest in a hierarchy of segregation models, with two-dimensional hyperbolic segregation theory (Savage \& Lun 1988; Gray \& Thornton 2005; Thornton et al. 2006) providing more detailed information about how unstably stratified particles readjust into a stable configuration (Gray et al. 2006; Shearer et al. 2008) and are recirculated in the flow (Thornton \& Gray 2008; Gray \& Ancey 2009) and, at the most complex level, the parabolic segregation-remixing equation (Dolgunin \& Ukolov 1995; Gray \& Chugunov 2006), which is able to reproduce the diffuse concentration profiles of steady-state bi-disperse particle dynamics simulations. The depth-averaged theory is appropriate for flows in which the large and small particles segregate rapidly from one another, and it has two key advantages. Firstly, one spatial dimension is removed from the problem, which makes it much easier to calculate the solution, and secondly, it is not necessary to know the precise values of the non-dimensional parameters $q$ and $D$ required by the full theory, since the transport equation is independent of them.

Although the one-dimensional transport equation has only a relatively simple representation of the particle size distribution, it does a surprisingly good job of capturing the essence of two-dimensional solutions to hyperbolic segregation theory. In particular, the equation for the inversely graded interface (3.2) between the large and small particles prior to breaking is exactly the same as equation (4.20) that Gray et al. (2006) derived for the full theory. Moreover, once the interface breaks a shock forms, whose speed (3.9) is precisely the same as Thornton \& Gray (2008) calculated in their equation (2.25) for the speed of the breaking size segregation wave. The net transport of large particles towards the flow front is therefore the same in the two theories, with a shock replacing the breaking size segregation wave as shown in figures 5 and 7. In $\S 5$ it was shown that the theory automatically captures the accumulation and recirculation of large particles at a flow front, which is crucial for the development of bouldery flow fronts in geophysical mass flows. When this process is combined with basal deposition, in $\S 4.1$, steadily travelling waves can develop that deposit all the large particles that reach the flow front. Intriguingly, even though the avalanche is inversely graded, the caterpillar track motion of the avalanche in this two-dimensional flow implies that the deposited grains are normally graded. This contrasts strongly with avalanches that are brought to rest by granular bores, in $\S 4.2$, which preserve the inversely graded stratified layers in the deposit. An inversely graded avalanche may therefore produce diametrically opposite final gradings, dependent on how the grains were brought to rest, which has important implications for the inferences that geologists can draw from sedimentary and pyroclastic flow deposits.

Large particle transport theory fits naturally into the depth-averaged framework of existing avalanche models and opens up the realistic possibility of coupling the evolving particle size distribution to the bulk dynamics. We may therefore envisage a new generation of avalanche models that are able to capture 'segregation-mobility' feedback effects. In one dimension, this would result in a system of three equations: the depth-averaged mass balance equation, the large particle transport equation and the depth-averaged downslope momentum balance equation, which in dimensional form are

$$
\frac{\partial h}{\partial t}+\frac{\partial}{\partial x}(h \bar{u})=-d,
$$




$$
\begin{gathered}
\frac{\partial \eta}{\partial t}+\frac{\partial}{\partial x}(\eta \bar{u})-\frac{\partial}{\partial x}\left((1-\alpha) \bar{u} \eta\left(1-\frac{\eta}{h}\right)\right)=-\phi_{b} d, \\
\frac{\partial}{\partial t}(h \bar{u})+\frac{\partial}{\partial x}\left(F h \bar{u}^{2}\right)+\frac{\partial}{\partial x}\left(\frac{1}{2} K g \cos \zeta h^{2}\right)=h S-u_{b} d,
\end{gathered}
$$

where $\phi_{b}$ and $u_{b}$ are the concentration and downslope velocity at the base of the flow and the source term

$$
S=g \cos \zeta\left(\tan \zeta-\frac{u}{|u|} \mu-\frac{\partial b}{\partial x}\right) .
$$

The coupling can be achieved by making (i) the basal friction $\mu$, (ii) the basal deposition $d$, (iii) the velocity shape profile $F$ or (iv) the earth pressure coefficient $K$, dependent on the local composition of the flow. The simplest coupling is through a composition-dependent friction coefficient $\mu$, which represents changes in friction as the proportion of large and small grains varies. The differential friction may arise because the grains have different surface properties, or shapes, e.g. small spheres mixed with larger irregular grains. Very little is known about the friction of such mixtures, and there are some surprising effects (e.g. Félix \& Thomas 2004; Goujon et al. 2007) even for large and small spheres on a rough slope. Another means of coupling is through the velocity shape factor $F$. For the linear velocity profile (2.17) used in this paper, $F=1+(1-\alpha)^{2} / 3$. However, it should be noted that if the value of $\alpha$ changes with $\eta$, then this modifies both the shape factor $F$ in (6.3) as well as the shear rate in (6.2). For more complex nonlinear velocity fields the flux function $h \overline{\phi u}$ in the depth-integrated segregation equation (2.14) must therefore be recomputed, which will yield a modified form for the large particle transport equation (6.2). The transport equation also generalizes easily to two dimensions, yielding a system of four equations, that should be able to capture the fingering effects of Pouliquen et al. (1997) by building-in frictional feedback. More complex couplings are needed for debris flows (Iverson 2003), where there are additional density effects, as well as feedback from the particle size distribution on the evolving internal pore pressure, which in turn modifies the basal friction.

This research was supported by NERC grant NE/E003206/1. N.G. also acknowledges support from an EPSRC Advanced Research Fellowship GR/S50052/ $01 \&$ GR/S50069/01.

Supplementary movies are available at journals.cambridge.org/flm.

\section{REFERENCES}

Abramowitz, M. \& Stegun, I. 1970 Handbook of Mathematical Functions, 9th edn, 3.3.7. Dover.

Aranson, I. S., Malloggi, F. \& Clement, E. 2006 Transverse instability in granular flows down an incline. Phys. Rev. E 73, 050302(R).

Bartelt, P. \& MCARdell, B. W. 2009 Granulometric investigations of snow avalanches. J. Glaciol. 55 (193), 829-833.

BaXter, J., TüzÜN, U., Heyes, D., Hayati, I. \& Fredlund, P. 1998 Stratification in poured granular heaps. Nature 391, 136.

Bertran, P. 2003 The rock-avalanche of February 1995 at Claix (French Alps). Geomorphology 54, 339-346.

Calder, E. S., Sparks, R. S. J. \& Gardeweg, M. C. 2000 Erosion, transport and segregation of pumice and lithic clasts in pyroclastic flows inferred from ignimbrite at Lascar Volcano, Chile. J. Volcanol. Geotherm. Res. 104, 201-235. 
Chadwick, P. 1999 Continuum Mechanics: Concise Theory and Problems. Dover.

Costa, J. E. \& Williams, G. 1984 Debris flow dynamics. Open-File Rep., 84-606 (videotape). US Geological Survey

Cui, X., Gray, J. M. N. T. \& Johannesson, T. 2007 Deflecting dams and the formation of oblique shocks in snow avalanches at Flateyri, Iceland. J. Geophys. Res. 112, F04012.

Dolgunin, V. N. \& Ukolov, A. A. 1995 Segregation modelling of particle rapid gravity flow. Powder Technol. 83, 95-103.

Doyle, E. E., Huppert, H. E., Lube, G., Mader, H. M. \& Sparks, R. S. 2007 Static and flowing regions in granular collapses down channels: insights from a sedimenting shallow water model. Phys. Fluids 19, 106601.

FÉLix, G. \& Thomas, N. 2004 Relation between dry granular flow regimes and morphology of deposits: formation of levees in pyroclastic deposits. Earth Planet. Sci. Lett. 221, 197-213.

Golick, L. A. \& Daniels, K. E. 2009 Mixing and segregation rates in sheared granular materials. Phys. Rev. E 80, 042301.

Goujon, C., Dalloz-Dubrujeaud, B. \& Thomas, N. 2007 Bidisperse granular avalanches on inclined planes: a rich variety of behaviours. Eur. J. Phys. E 23, 199-215.

Grasselli, Y. \& Herrmann, H. J. 1997 On the angles of dry granular heaps. Physica A 246, 301-312.

GraY, J. M. N. T. 2001 Granular flow in partially filled slowly rotating drums. J. Fluid Mech. 441, $1-29$.

Gray, J. M. N. T. \& Ancey, C. 2009 Segregation, recirculation and deposition of coarse particles near two-dimensional avalanche fronts. J. Fluid Mech. 629, 387-423.

Gray, J. M. N. T. \& Chugunov, V. A. 2006 Particle-size segregation and diffusive remixing in shallow granular avalanches. J. Fluid Mech. 569, 365-398.

Gray, J. M. N. T. \& CUI, X. 2007 Weak, strong and detached oblique shocks in gravity-driven granular free-surface flows. J. Fluid Mech. 579, 113-136.

Gray, J. M. N. T. \& Hutter, K. 1997 Pattern formation in granular avalanches. Contin. Mech. Thermodyn. 9, 341-345.

Gray, J. M. N. T., Shearer, M. \& Thornton, A. R. 2006 Time-dependent solutions for particle-size segregation in shallow granular avalanches. Proc. R. Soc. A 462, 947-972.

Gray, J. M. N. T., TaI, Y. C. \& Noelle, S. 2003 Shock waves, dead-zones and particle-free regions in rapid granular free-surface flows. J. Fluid Mech. 491, 161-181.

Gray, J. M. N. T. \& Thornton, A. R. 2005 A theory for particle size segregation in shallow granular free-surface flows. Proc. R. Soc. A 461, 1447-1473.

Gray, J. M. N. T., Wieland, M. \& Hutter, K. 1999 Free surface flow of cohesionless granular avalanches over complex basal topography. Proc. R. Soc. A 455, 1841-1874.

Grigorian, S. S., Eglit, M. E. \& Iakimov, IU. L. 1967 New state and solution of the problem of the motion of snow avalanche. Snow Avalanches Glaciers, Trans. Vysokogornogo Geofiz. Inst. 12, 104-113.

Gruber, U. \& Bartelt, P. 2007 Snow avalanche hazard modelling of large areas using shallow water numerical methods and GIS. Environ. Model. Softw. 22 (10), 1472-1481.

Herrmann, H. J. 1998 On the shape of a sandpile. In Physics of Dry Granular Media (ed. H. J. Herrmann, J.-P. Hovi \& S. Luding), NATO ASI Series, pp. 697-702. Kluwer Academic.

Hill, K. M., Gioia, G. \& Amaravadi, D. 2004 Radial segregation patterns in rotating granular mixtures: waviness selection. Phys. Rev. Lett. 93 (224301), 1-4.

IVErson, R. M. 1997 The physics of debris-flows. Rev. Geophys. 35, 245-296.

IVERSON, R. M. 2003 The debris-flow rheology myth. In Debris-Flow Hazards Mitigation: Mechanics, Prediction and Assessment (ed. D. Rickenmann \& C. L. Chen), pp. 303-314. Millpress.

Iverson, R. M. \& Denlinger, R. P. 2001 Flow of variably fluidized granular masses across threedimensional terrain. Part 1. Coulomb mixture theory. J. Geophys. Res. 106 (B1), 553-566.

Iverson, R. M. \& Vallance, J. W. 2001 New views of granular mass flows. Geology 29 (2), 115-118.

Jesuthasan, N., Baliga, B. R. \& Savage S. B. 2006 Use of particle tracking velocimetry for measurements of granular flows: review and application. KONA 24, 15-26.

Jiang, G.-S., Levy, D., Lin, C.-T., Osher, S. \& TADMOR, E. 1998 High-resolution nonoscillatory central schemes with non-staggerred grids for hyperbolic conservation laws. SIAM J. Numer. Anal. 35 (6), 2147-2168. 
JiANG, G.-S. \& TADMOR, E. 1998 Nonoscillatory central schemes for multidimensional hyperbolic conservation laws. SIAM J. Sci. Comput. 19 (6), 1892-1917.

Johanson, J. R. 1978 Particle segregation ... and what to do about it. Chem. Engng, 183-188.

Jomelli, V. \& Bertran, P. 2001 Wet snow avalanche deposits in the French Alps: structure and sedimentology. Geografis. Annal. A 83 (1/2), 15-28.

Jop, P., Forterre, Y. \& Pouliquen, O. 2006 A constitutive relation for dense granular flows. Nature 44, 727-730.

Khakhar, D. V., McCarthy, J. J. \& Ottino, J. M. 1999 Mixing and segregation of granular materials in chute flows. Chaos 9, 594610.

Lajeunesse, E., Mangeney-Castelnau, A. \& Vilotte, J. P. 2004 Spreading of a granular mass on a horizontal plane. Phys. Fluids 16, 2371.

Linares-Guerrero, E., Goujon, C. \& Zenit, R. 2007 Increased mobility of bidisperse granular flows. J. Fluid Mech. 593, 475-504.

Lube, G., Huppert, H. E., Sparks, R. S. J. \& Hallworth, M. A. 2004 Axisymmetric collapses of granular columns. J. Fluid Mech. 508, 175-199.

Makse, H. A., Havlin, S., King, P. R. \& Stanley, H. E. 1997 Spontaneous stratification in granular mixtures. Nature 386, 379-382.

Mangeney, A., Bouchut, F., Thomas, N., Vilotte, J. P. \& Bristeau, M. O. 2007 Numerical modelling of self-channeling granular flows and of their levee-channel deposits. J. Geophys. Res. 112, F02017.

Mcintyre, M., Rowe, E., Shearer, M., Gray, J. M. N. T. \& Thornton, A. R. 2007 Evolution of a mixing zone in granular avalanches. $A M R X \mathbf{2 0 0 7}$, abm008.

Middleton, G. V. 1970 Experimental studies related to problems of flysch sedimentation. In Flysch Sedimentology in North America (ed. J. Lajoie), pp. 253-272. Business and Economics Science Ltd.

Middleton, G. V. \& Hampton, M. A. 1976 Subaqueous sediment transport and deposition by sediment gravity flows. In Marine Sediment Transport and Environmental Management (ed. D. J. Stanley \& D. J. P. Swift), pp. 197-218. Wiley.

Nessyahu, H. \& TADmoR, E. 1990 Non-oscillatory central differencing for hyperbolic conservation laws. J. Comput. Phys. 87, 408-463.

Phillips, J. C., Hogg, A. J., Kerswell, R. R. \& Thomas, N. H. 2006 Enhanced mobility of granular mixtures of fine and coarse particles. Earth Planet. Sci. Lett. 246, 466-480.

Pierson, T. C. 1986 Flow behaviour of channelized debris flows, Mount St. Helens, Washington. In Hillslope Processes (ed. A. D. Abrahams), pp. 269-296. Allen and Unwin.

Pitman, E. B., Nichita, C. C., Patra, A., Bauer, A., Sheridan, M. \& Bursik, M. 2003 Computing granular avalanches and landslides. Phys. Fluids 15 (12), 3638-3646.

Pouliquen, O. 1999a Scaling laws in granular flows down rough inclined planes. Phys. Fluids 11 (3), 542-548.

Pouliquen, O. $1999 \mathrm{~b}$ On the shape of granular fronts down rough inclined planes. Phys. Fluids $11(7), 1956-1958$.

Pouliquen, O., Delour, J. \& Savage, S. B. 1997 Fingering in granular flows. Nature 386, 816-817.

Pouliquen, O. \& Forterre, Y. 2002 Friction law for dense granular flows: application to the motion of a mass down a rough inclined plane. J. Fluid Mech. 453, 133-151.

Pouliquen, O. \& Vallance, J. W. 1999 Segregation induced instabilities of granular fronts. Chaos 9 (3), 621-630.

Rognon, P. G., Roux, J. N., NaAim, M. \& Chevoir, F. 2007 Dense flows of bidisperse assemblies of disks down an inclined plane. Phys. Fluids 19, 058101.

Savage, S. B. \& Hutter, K. 1989 The motion of a finite mass of granular material down a rough incline. J. Fluid Mech. 199, 177-215.

Savage, S. B. \& Lun, C. K. K. 1988 Particle size segregation in inclined chute flow of dry cohesionless granular solids. J. Fluid Mech. 189, 311-335.

Shearer, M. Gray, J. M. N. T. \& Thornton, A. R. 2008 Stable solutions of a scalar conservation law for particle-size segregation in dense granular avalanches. Eur. J. Appl. Math. 19, 61-86.

Tai, Y.-C., Noelle, S., Gray, J. M. N. T. \& Hutter, K. 2002 Shock-capturing and front-tracking methods for granular avalanches. J. Comput. Phys. 175, 269-301.

Thornton, A. R., Gray, J. M. N. T. \& HogG, A. J. 2006 A three-phase mixture theory for particle size segregation in shallow granular free-surface flows. J. Fluid Mech. 550, 1-25. 
Thornton, A. R. \& Gray, J. M. N. T. 2008 Breaking size-segregation waves and particle recirculation in granular avalanches. J. Fluid Mech. 596, 261-284.

Vallance, J. W. \& SaVAGE, S. B. 2000 Particle segregation in granular flows down chutes. In IUTAM Symposium on Segregation in Granular Materials (ed. A. D. Rosato \& D. L. Blackmore), pp. 31-51. Kluwer.

Whitham, G. B. 1974 Linear and Nonlinear Waves. Wiley.

Wieland, M., Gray, J. M. N. T. \& Hutter, K. 1999 Channelised free surface flow of cohesionless granular avalanches in a chute with shallow lateral curvature. J. Fluid Mech. 392, 73-100.

Williams, S. C. 1968 The mixing of dry powders. Powder Technol. 2, 13-20.

Zuriguel, I., Gray, J. M. N. T., Peixinho, J. \& Mullin, T. 2006 Pattern selection by a granular wave in a rotating drum. Phys. Rev. E 73 (061302), 1-4. 


\title{
E R R A T U M
}

\section{Large particle segregation, transport and accumulation in granular free-surface flows - ERRATUM}

\author{
J. M. N. T. GRA Y AND B. P. KOKELAAR
}

(Received 3 June 2010)

doi:10.1017/S02211201000011X, Published by Cambridge University Press 19 May 2010

The Publishers apologise to the authors and readers for the following errors which occurred in Gray \& Kokelaar (2010).

(a) ON P. 116 THE JUMP BRACKETS ARE MISSING:

... At such discontinuities $\eta$ satisfies the jump condition (see e.g. Chadwick 1976; Gray, Shearer \& Thornton 2006, for a general derivation)

$$
\llbracket \eta\left(\bar{u}-v_{n}\right) \rrbracket=\llbracket(1-\alpha) \bar{u} \eta\left(1-\frac{\eta}{h}\right) \rrbracket,
$$

where $v_{n}$ is the normal speed of the shock and the jump bracket $\llbracket f \rrbracket=f_{2}-f_{1}$ is the difference of the enclosed quantity on the forward and rearward sides of the shock (denoted by the subscripts 2 and 1, respectively).

(b) ON P. 126 ALSO THE JUMP BRACKETS ARE MISSING:

... These can be summarized by the relations

$$
\begin{aligned}
\llbracket h\left(\bar{u}-v_{n}\right) \rrbracket & =0, \\
\llbracket h \bar{u}\left(\bar{u}-v_{n}\right) \rrbracket+\llbracket \frac{1}{2} h^{2} \varepsilon \cos \zeta \rrbracket & =0, \\
\llbracket \eta\left(\bar{u}-v_{n}\right) \rrbracket-\llbracket(1-\alpha) \bar{u} \eta(1-\eta / h) \rrbracket & =0,
\end{aligned}
$$

where the velocity magnitude is assumed to scale as $U=\sqrt{g L}$.

\section{REFERENCES}

Chadwick, P. 1976 Continuum Mechanics. Concise Theory and Problems, 187 pp. George Allen \& Unwin (republished Dover 1999).

Gray, J. M. N. T. \& KoKelaAR, B. P. 2010 Large particle segregation, transport and accumulation in granular free-surface flows. J. Fluid Mech. 652, 105-137.

Gray, J. M. N. T., Shearer, M. \& Thornton, A. R. 2006 Time-dependent solutions for particle-size segregation in shallow granular avalanches. Proc. R. Soc. A 462, 947-972. 